\title{
Ultralong-range molecule engineering via Rydberg dressing
}

\author{
Jia Wang (10 \\ Centre for Quantum and Optical Science, Swinburne University of Technology, Melbourne, Victoria 3122, Australia \\ Robin Côté \\ Physics Department, University of Massachusetts Boston, Boston, Massachusetts 02125, USA \\ and Department of Physics, University of Connecticut, Storrs, Connecticut 06269, USA
}

(Received 20 November 2019; accepted 27 February 2020; published 8 April 2020)

\begin{abstract}
In 2000, three pioneering papers launched a new era in Rydberg physics. One predicted the blockade mechanism where extremely large Rydberg-Rydberg interactions only allow a single excitation in a given volume. A second envisioned how strong long-range interactions between ground-state atoms could be induced via admixing with Rydberg character with dressing lasers. The third foresaw the existence of a new type of molecules bound by the Rydberg electron, namely, ultralong-range Rydberg molecules (URMs), sometimes known as trilobitelike molecules. We predict a new molecular binding mechanism between ground-state atoms based on the combination of aspects of each feature. By using lasers to dress interactions with a URM state, within a blockade volume, we find that pairs of atoms can be bound in potential wells at separations of thousands of Bohr radii. We show how the wells' properties can be tailored by laser fields. The bound levels produced have unique properties, such as very long bond length and much longer lifetimes than their URM "parents," and, contrary to standard molecular levels, they can be adjusted easily by the appropriate choice of laser parameters or Rydberg dressing state. Furthermore, the spatial orientation and even the geometry of those molecules can be designed and controlled. This approach could also be employed to generate correlated pairs, allowing to engineer atoms' spatial distributions to explore many-body dynamics in ultracold samples.
\end{abstract}

DOI: 10.1103/PhysRevResearch.2.023019

\section{INTRODUCTION}

A key contribution of ultracold systems is the unprecedented control over interactions. For example, advances in cooling and trapping of atomic samples allow temperatures in the nanokelvin regime and densities in the $10^{12}-10^{15} \mathrm{~cm}^{-3}$ range, providing an ideal platform to explore quantum phenomena such as Bose-Einstein condensation (BEC) [1-3]. Magnetically tunable Feshbach resonances [4-6] to control the interactions between atoms ultimately led to a variety of studies, from many-body physics such as the BEC-BCS crossover regime [7-9] or soliton physics [10,11], to few-body physics such as the observation of Efimov states [12] or the universality of three-body physics near thresholds [13-15]. Similarly, employing periodic potentials like optical lattices [16] allows investigations of quantum phase transitions [17] as well as quantum simulations $[18,19]$.

Another tool to "engineer" interactions relies on ultracold Rydberg atoms and their extreme properties, which scale rapidly with their principal quantum number $n$, such as their radius and dipole moment, $n^{2}$, polarizability, $n^{7}$, energy level spacing, $n^{-3}$, or lifetime, $n^{3}$ [20]. In 2000, three pioneering

Published by the American Physical Society under the terms of the Creative Commons Attribution 4.0 International license. Further distribution of this work must maintain attribution to the author(s) and the published article's title, journal citation, and DOI. articles predicted the blockade mechanism [21], Rydberg dressing of atoms interactions [22], and ultralong-range Rydberg molecules (URMs) such as trilobite states [23], following the initial studies of resonant excitations [24] and excitation transfer [25]. Since then, entanglement using strong dipoledipole interactions was realized [26] and generalization of the Rydberg blockade to ensembles was proposed [27]. The implementation of Rydberg dressing is discussed in detail in Ref. [28]. While Rydberg dressing faces several technical difficulties such as dissipation and spontaneous scattering, it has been recently observed in optical tweezers [29] and optical lattices [30] after some earlier efforts to overcome these difficulties [31]. Applications of Rydberg dressing have been proposed to achieve three-dimensional self-trapping of matter waves [32], to tune chemical reactions [33], and to model frustrated quantum magnets [34], and more recently Rydberg dressing was achieved in a Ramsey interferometer and electrometer experiment [35]. Other approaches to achieve Rydberg dressing such as using Förster resonances have also been proposed [36]. Rydberg atoms have also found applications in quantum information and quantum computing [37], in quantum optics [38], and in chemical systems with the emergence of new types of bonds, such as the URM [23] or the even more extended macrodimers [39-41] recently detected [42-44].

The URM states arise when the effective interaction of the highly excited electron of a Rydberg atom colliding with a ground-state atom is attractive. Originally formulated using a Fermi pseudopotential model [45] for $s$-wave scattering [23], it has been extended to $p$-waves [46,47] and is known as 
butterfly states. The initial observation of these URM states in $\mathrm{Rb}$ [48] has been followed by investigations of their properties in $\mathrm{Rb}$ [49-52], Cs [53,54], and Sr [55]. Recently, predictions [56] and observations [57] of optical Feshbach resonances (OFRs) based on coupling to URM states have been made.

In this work, we describe how we can control the interaction between ground-state atoms by partially imprinting the scattering of a Rydberg electron between them. We explore how a new binding mechanism between ground-state atoms, based on Rydberg dressing, can lead to ultralong-range molecules, which we call ultralong-range Rydberg-dressed molecules (URdMs). We show that by using far-detuned lasers to couple a small but adjustable Rydberg component to the ground-state atom, localized long-range potential wells that support molecular bound levels can be created. These potential wells and URdM states can, in some cases, possess very different character than their parent URM states. We also note that our system, although seemingly similar to OFRs presented in Refs. [56,57], differs in subtle but important ways from OFRs as we detail later.

We apply this concept to $\mathrm{Rb}$, as it is the workhorse of ultracold Rydberg physics, and consider dressing by $s, p$, and $d$ Rydberg states using different photon polarizations. We find that each Rydberg-dressing type can sustain bound levels, with linear polarization leading to larger binding energies, and show that the spatial arrangement of this new type of molecule can be controlled via laser polarizations. We also extend the treatment from diatomic to polyatomic systems, where even the geometry of these molecules can be engineered.

\section{MODEL}

We first describe the dressing schemes by $s, p$, and $d$ Rydberg states, followed a discussion of URM potential obtained from Fermi pseudopotentials. We then present how the Rydberg dressing of ground-state atoms using URMs leads to URdMs, first within a simplified treatment and second in a two-channel model treatment.

\section{A. Rydberg dressing}

Rydberg dressing is currently a very active field of investigation that has been extensively explored both theoretically and experimentally. The basic idea can be understood via a simple two-level atom model, with its ground state $|g\rangle$ coupled to a Rydberg state $|e\rangle$ by optical fields. In particular, we consider specific Rydberg states $|e\rangle \equiv\left|n \ell j m_{j}\right\rangle$ of an alkalimetal atom with sizable quantum defect $\delta_{n \ell j}$ corresponding to the $n s_{j}$ state $(\ell=0), n p_{j}$ state $(\ell=1)$, and $n d_{j}$ state $(\ell=2)$ $[23,58]$. Here, $n$ is the principle quantum number, $\ell(j)$ is the orbital (total) angular momentum of the Rydberg electron, and $m_{\ell}\left(m_{j}\right)$ denotes the projection of $\ell(j)$ on the quantization axis defined by the dressing laser polarization. The $\operatorname{spin} s=1 / 2$ of the Rydberg electron has a projection $m_{s}$ on the same axis, and we assume a small bias field along that quantization axis, so that ground-state atoms with $\ell_{g}=0$ and spin $s_{g}=1 / 2$ can be prepared in spin-up projection $\left(m_{j_{g}}=m_{s_{g}}=1 / 2\right)$ states, e.g., $|g\rangle=\left|5 s_{1 / 2},+\frac{1}{2}\right\rangle$ for ${ }^{87} \mathrm{Rb}$.

The optical fields are described by a Rabi frequency $\Omega$, a detuning $\Delta$, and a polarization $\sigma$. A one-photon scheme

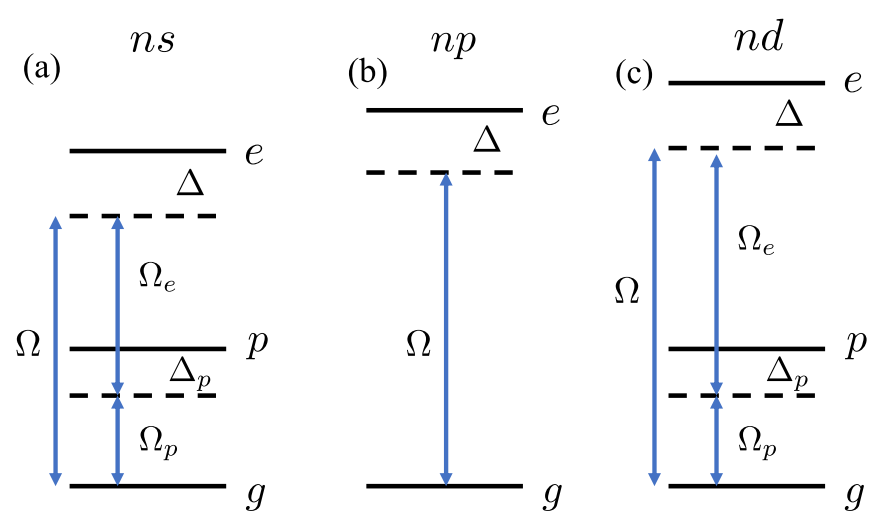

FIG. 1. Sketch of excitation schemes in a single-atom basis. The ground state is coupled to the Rydberg $n p$ ( $n s$ or $n d$ ) state by onephoton (two-photon) schemes with (effective) Rabi frequency $\Omega$ and detuning $\Delta$.

characterized by these parameters can accomplish the dressing with a Rydberg $n p_{j}$ state directly as depicted in Fig. 1, while two-photon excitations are required for $n s$ or $n d$ states. A first laser couples $|g\rangle$ to an intermediate $n^{\prime} p$ level (where $n^{\prime}=5$ for ${ }^{87} \mathrm{Rb}$ ), with Rabi frequency $\Omega_{p}$, detuning $\Delta_{p}$, and polarization $\sigma_{p}$, while a second laser couples this intermediate level to $|e\rangle$ with Rabi frequency $\Omega_{e}$ and polarization $\sigma_{e}$, leading to a total detuning $\Delta$ from $e$ and an effective total polarization $\sigma=\sigma_{p}+\sigma_{e}$. For large enough $\Delta_{p}$, the intermediate state can be eliminated, leading to an effective Rabi frequency $\Omega=\Omega_{p} \Omega_{e} / 2\left|\Delta_{p}\right|$. The polarization of the dressing lasers defines the polarization axis as our quantization axis and determines $m_{j}=m_{j_{g}}+\sigma$. As discussed later, the selected $m_{j}$ ultimately leads to the angular momentum dependence of the dressed interaction in the laboratory frame.

For a single atom, the Hamiltonian can be written as a twolevel system $\Delta|e\rangle\langle e|+\frac{\Omega}{2}(|g\rangle\langle e|+$ H.c. $)$ within the rotatingwave approximation. The dressed ground state is therefore given by $\alpha_{1}|g\rangle+\beta_{1}|e\rangle$, where $b_{1} \equiv\left|\beta_{1}\right|^{2} \approx|\Omega / 2 \Delta|^{2}$ in the far-detuned limit $\Omega \ll \Delta$ describes the probability to find the atom in Rydberg states $|e\rangle$. The subscript 1 is used to explicitly indicate that the coefficients are for a one-atom dressing case. Such a state would only have a fraction of the Rydberg character and a much longer lifetime determined by the mixing parameter $b_{1}$. In particular, the interactions between the Rydberg-dressed atoms is approximately proportional to $b_{1}$, which is the underlying physics of URdMs studied in this work.

\section{B. Fermi pseudopotential and URM potential}

Another important ingredient in this work is the URM potential based on the Fermi pseudopotential description of electron-atom scattering originated from Fermi's idea [45]. Following Eq. (9) of Ref. [58], the Fermi pseudopotential that includes all the spin effects and the singlet (triplet) $s$-wave ( $p$-wave) scattering is given by (in atomic units)

$$
\begin{aligned}
\hat{V}_{F}\left(\mathbf{r}_{e}, \mathbf{R}\right)= & \sum_{J, L, S} \sum_{M_{S}, M_{S}^{\prime}, M_{J}} \mathcal{A}_{S L}^{J} \mathcal{C}_{M_{S} M_{S}^{\prime}}^{J M_{J}} \\
& \times\left|S, M_{S}\right\rangle\left\langle S, M_{S}^{\prime}\right| \overleftarrow{\nabla}_{M_{L}}^{L} \delta\left(\mathbf{r}_{e}-\mathbf{R}\right) \vec{\nabla}_{M_{L}^{\prime}}^{L}
\end{aligned}
$$


where $\quad \mathcal{C}_{M_{S} M_{S}^{\prime}}^{J M_{J}}=C_{L M_{L}, S M_{S}}^{J M_{J}} C_{L M_{L}^{\prime}, S M_{S}^{\prime}}^{J M_{J}}$ with $\quad M_{L}=M_{J}-M_{S}$, $M_{L}^{\prime}=M_{J}-M_{S}^{\prime}$, and $C_{L M_{L}, S M_{S}}^{J M_{J}}$ being the Clebsch-Gordan coefficients. Here, $\mathcal{A}_{S L}^{J}=(2 L+1) 2 \pi a_{L S}^{J}\left(k_{R}\right)$ depends on the momentum $k_{R}$ and is given in terms of the energy-dependent scattering length ( $s$ wave with $L=0$ ) or volume ( $p$ wave with $L=1) a_{S L}^{J}\left(k_{R}\right)$ corresponding to a singlet $(S=0)$ or triplet $(S=1)$ interaction between a quasifree electron and an atom including the spin-orbit splitting characterized by the total angular momentum $J$. Correspondingly, $M_{J}, M_{L}$, and $M_{S}$ are the projections of $J, L$, and $S$. Here, $\mathbf{R}$ and $\mathbf{r}_{e}$ are the position of the neutral atom and the Rydberg electron with respect to the ion core, and the relative electron-atom semiclassical momentum $k_{R}=\sqrt{2 / R-1 / n_{H}^{2}}$ depends on $R$ with $n_{H}$ being the principal quantum number of the nearest hydrogenic manifold [23,58]. The scattering length and volume are given in term of the scattering phase shift $\eta_{S L}^{J}\left(k_{R}\right)$ via $k_{R}^{2 L+1} a_{S L}^{J}\left(k_{R}\right)=-\tan \eta_{S L}^{J}\left(k_{R}\right)$ [46].

A commonly adopted simplified treatment for a qualitative understanding is to include $s$-wave scattering $(L=0)$ only. Moreover, we consider alkali-metal atoms such as ${ }^{87} \mathrm{Rb}$, for which singlet $(S)$ scattering lengths are positive $\left(a_{s}^{S} \equiv\right.$ $\left.a_{L=0, S=0}^{J=0}>0\right)$ and triplet $(T)$ scattering lengths are negative $\left(a_{s}^{T} \equiv a_{L=0, S=1}^{J=1}<0\right)[46,59,60]$. Since only negative electronatom scattering lengths lead to URM trilobitelike wells [23], we keep exclusively the triplet $s$-wave scattering described by $a_{s}^{T}$, so that

$$
\hat{V}_{F}^{\mathrm{app}}\left(\mathbf{r}_{e}, \mathbf{R}\right) \simeq 2 \pi a_{s}^{T}\left(k_{R}\right) \delta\left(\mathbf{r}_{e}-\mathbf{R}\right) \sum_{M_{S}}\left|1, M_{S}\right\rangle\left\langle 1, M_{S}\right|
$$

is our approximate pseudopotential. This approximate treatment, qualitative for alkali-metal atoms like ${ }^{87} \mathrm{Rb}$, is more quantitative for bosonic alkaline-earth elements, such as ${ }^{86} \mathrm{Sr}$ or ${ }^{88} \mathrm{Sr}$, which possess no hyperfine structure and negligible $p$-wave scattering [61], in addition to having only one doublet $S=\frac{1}{2}$ spin state and corresponding scattering length.

Under this approximation, the two-body interaction operator between atoms $A$ and $B$ is given by $\hat{V}_{2 b}^{\text {app }}=$ $\sum_{\mathbf{r}_{e}^{A}} \hat{V}_{F}^{\text {app }}\left(\mathbf{r}_{e}^{A}, \mathbf{R}\right)\left|\mathbf{r}_{e}^{A}\right\rangle_{A}\left\langle\mathbf{r}_{e}^{A}|\otimes| 5 s_{1 / 2}\right\rangle_{B}\left\langle 5 s_{1 / 2}\right|+(A \Leftrightarrow B)$. The Born-Oppenheimer potential between a ground-state atom and an atom in a specific Rydberg state selected by a laser can be obtained as a first-order perturbation $V_{\mathrm{URM}}(\mathbf{R})=$ $\left\langle g e\left|\hat{V}_{2 b}^{\mathrm{app}}\right| g e\right\rangle$, which gives

$$
V_{\mathrm{URM}}(\mathbf{R})=2 \pi a_{s}^{T}\left(k_{R}\right) \mathcal{R}_{v \ell}^{2}(R) \Theta(\hat{\mathbf{R}}),
$$

where $v=n-\delta_{n \ell j}$ is the effective principal quantum number with $\delta_{n \ell j}$ being the quantum defect. The radial wave function $\mathcal{R}_{\nu \ell}$ is the Coulomb function with vanishing boundary condition as $R \rightarrow \infty$, i.e., $\mathcal{R}_{v \ell}(R)=W(R, v, \ell) / R$ with $W(R, v, \ell)$ being the "energy-normalized" Whittaker function [62]. Contrary to the URM potentials in previous studies such as Ref. [23] (where the Rydberg state is not coherently coupled to the ground state by laser), Eq. (3) shows an angular dependence

$$
\Theta(\hat{\mathbf{R}})=\sum_{m_{\ell}} c_{m_{\ell}}^{2}\left|Y_{\ell m_{\ell}}(\hat{\mathbf{R}})\right|^{2},
$$

since the $m_{j}$ of the Rydberg state here is coherently selected by the laser polarization, where $Y_{\ell m_{\ell}}$ is the usual spherical

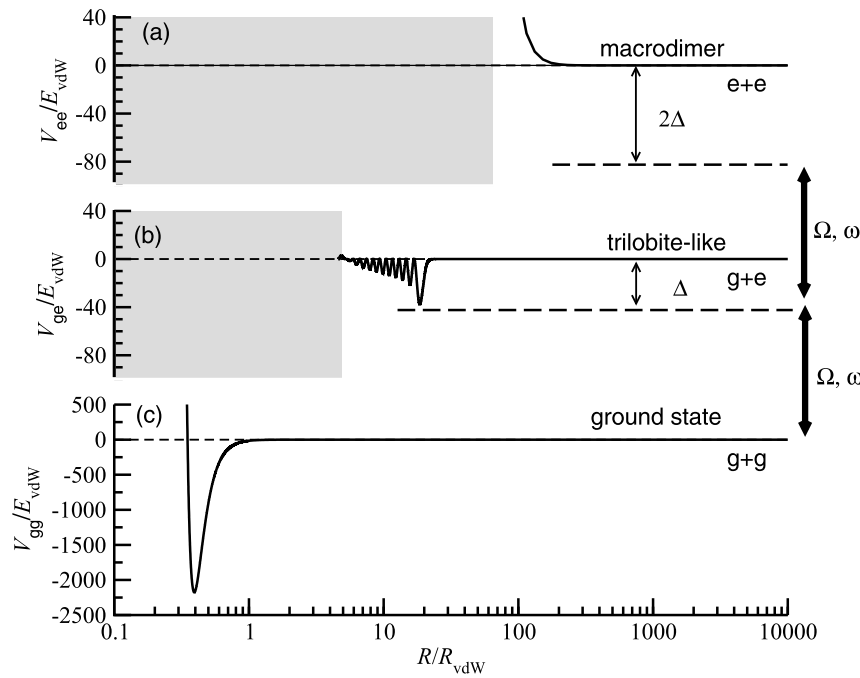

FIG. 2. Sketch of interaction $\left(E_{\mathrm{vdW}}=\hbar^{2} / m R_{\mathrm{vdW}}^{2}\right)$ between (a) two Rydberg atoms $(e+e)$, (b) a ground-state atom and a Rydberg atom $(g+e)$, and (c) two ground-state atoms $(g+g)$. These curves are weakly coupled by the dressing laser blue of frequency $\omega$ red-detuned by $\Delta$ from $g+e$ with Rabi frequency $\Omega$. The trilobitelike potential located near $10 R_{\mathrm{vdW}}$ is well separated from the groundstate molecular potential relevant below $R_{\mathrm{vdW}}$ and the macrodimer curve (important around $100 R_{\mathrm{vdW}}$ ); near the trilobitelike well, the $g+g$ interaction is essentially zero while the $e+e$ channel is within the Rydberg blockade region and can be neglected.

harmonic with angles $\quad \hat{\mathbf{R}} \equiv\left\{\theta_{R}, \phi_{R}\right\}, \quad$ and $\quad c_{m_{\ell}}=$ $C_{\ell m_{\ell}, s m_{s}}^{j m_{j}} C_{s m_{s}, s_{g} m_{s_{g}}}^{S M_{s}}$. Here, $C_{\ell m_{\ell}, s m_{s}}^{j m_{j}}$ is a Clebsch-Gordan coefficient, $M_{S}=m_{s}+m_{s_{g}}, \quad m_{s}=m_{j}-m_{\ell}, \quad m_{s_{g}}=1 / 2$ is selected as initial ground-state spin of atoms in the gas, $m_{j}=m_{s_{g}}+\sigma$ is determined by the polarization of the dressing lasers, and $S=1$ since we only consider triplet scattering. This angular dependence gives an anisotropic potential for $\ell>0$, with the direction determined by the laboratory-frame quantization axis defined by the dressing laser's polarization. This anisotropy, as we show later, is essential for controlling the direction of molecular axis. We can also see that the potential still possesses azimuthal symmetry and is independent of $\phi_{R}$; therefore, we only need to calculate the potential at $\phi_{R}=0$. The form of $V_{\mathrm{URM}}$ also implies a reflection symmetry, i.e., $V_{\mathrm{URM}}(\mathbf{R})=V_{\mathrm{URM}}(-\mathbf{R})$.

\section{Simplified picture: Perturbation treatment}

We are now ready to study how to apply Rydberg dressing and the URM potential to manipulate interactions between Rydberg-dressed atoms. To investigate the interactions between Rydberg-dressed atoms, we consider two atoms in their ground state $|g\rangle$ weakly coupled by a laser to a specific excited Rydberg state $|e\rangle$. Such a system shows a well-defined separation of length scales between interactions correlated to the $g+g, g+e$, and $e+e$ asymptotes. Figure 2 sketches the relevant scales for $e$ corresponding to a Rydberg $n s$ state, where $n \approx 30$, with lasers characterized by Rabi frequency $\Omega$ and red-detuned by $\Delta$ from $e$. The $g+g$ interaction is only significant in the length scale of $R_{\mathrm{vdW}} ; R_{\mathrm{vdW}} \approx 82.58 \mathrm{Bohr}$ radii $\left(a_{0}\right)$ for ${ }^{87} \mathrm{Rb}$ is a length scale defined from the van der 
Waals interaction strength [4]. The $e+e$ interaction is dominated by dispersion interactions at a much larger distance $R>$ $100 R_{\mathrm{vdW}}$ associated with Rydberg macrodimers [39]. The effects of these long-range interactions have been observed as molecular resonances [63-65]. Dressing the $e+e$ interactions has been explored theoretically [22]. Here, we propose to manipulate long-range molecular bonds by dressing the $g+e$ trilobitelike interaction at intermediate distances.

The $g+e$ interaction, characterized by $V_{\mathrm{URM}}(\mathbf{R})$, dominates at intermediate distances around $10 R_{\mathrm{vdW}}$. At such separations, the doubly excited state $|e e\rangle$ corresponding to macrodimers can be safely omitted due to the now familiar Rydberg blockade mechanism. In addition, the antisymmetric state $|A\rangle \equiv \frac{1}{\sqrt{2}}(|g e\rangle-|e g\rangle)$ is uncoupled and can be ignored as well [66]. The Hamiltonian dictating the dynamics of the system can thus be described in the basis $|G\rangle \equiv|g g\rangle$ and $|S\rangle \equiv \frac{1}{\sqrt{2}}(|g e\rangle+|e g\rangle)$. Here, we use the notation $\left|a a^{\prime}\right\rangle \equiv$ $|a\rangle_{1} \otimes\left|a^{\prime}\right\rangle_{2}$ to mean that atom 1 is in state $|a\rangle$ and atom 2 is in $\left|a^{\prime}\right\rangle$.

We first introduce a qualitative description for alkali-metal atoms which considers the URM interaction induced by the Rydberg electron-atom scattering as a first-order correction, and apply the approximate two-body interaction operator $V_{2 b}^{\text {app }}$ from the previous section. A full treatment including $p$-wave scattering, electron-atom singlet channels, and spin-orbit splitting, as well as the effect of the hyperfine structures is also described later in Sec. III D. If we neglect the URM interactions first (and treat it as perturbation later), the effective twolevel Hamiltonian reads as $\Delta|S\rangle\langle S|+\frac{\Omega}{\sqrt{2}}(|S\rangle\langle G|+$ H.c. $)$. The dressed ground state is given by $\left|G^{*}\right\rangle=\alpha|G\rangle+\beta|S\rangle$, with $|\alpha|^{2}+|\beta|^{2}=1$ and $b \equiv|\beta|^{2}$. In the far-detuned limit $\Omega \ll \Delta$, the mixing parameter $b \approx|\Omega|^{2} / 2 \Delta^{2}=2 b_{1}$, where the factor of 2 indicates a collective enhancement. The first-order perturbative energy correction gives the Born-Oppenheimer (BO) potential for a pair of Rydberg-dressed atoms separated by $\mathbf{R}$, i.e., $U(\mathbf{R})=\left\langle G^{*}\left|\hat{V}_{2 b}^{\mathrm{app}}(\mathbf{R})\right| G^{*}\right\rangle=b V_{\mathrm{URM}}(\mathbf{R})$. The physical interpretation is clear and simple; the system has a probability $b$ to be in $e+g$, and hence only feels a fraction $b$ of the $e+g$ trilobite potential $V_{\mathrm{URM}}(\mathbf{R})$.

\section{Two-channel model treatment}

The perturbation treatment above assumes a weak dressing $(\Omega \ll \Delta)$. To account for stronger dressing, we adopt a twochannel treatment. The Hamiltonian describing the coupling between $g+g$ and $g+e$ then has a simple $2 \times 2$ matrix representation in the basis $|G\rangle$ and $|S\rangle$ [33], given by (in atomic units)

$$
H_{i}=\left[\begin{array}{lc}
V_{G}(\mathbf{R}) & \Omega / \sqrt{2} \\
\Omega^{*} / \sqrt{2} & \Delta+V_{\mathrm{URM}}(\mathbf{R})
\end{array}\right] .
$$

As discussed above and shown in Fig. 2, the interaction $V_{G}(\mathbf{R})$ between two ground-state atoms is shorter range and negligible where $V_{\mathrm{URM}}(\mathbf{R})$ is dominant, and thus can be set to zero in a simplified treatment. The lower eigenvalue $\mathcal{E}(\mathbf{R})$ obtained by diagonalizing $H_{i}$ at fixed $\mathbf{R}$ gives the BO potential energy surfaces (PESs) for a pair of Rydbergdressed atoms. Correspondingly, the normalized eigenvector $\left[\phi_{G}(\mathbf{R}), \phi_{S}(\mathbf{R})\right]^{\dagger}$ gives a local mixing parameter $b(\mathbf{R})=$
$\left|\phi_{S}(\mathbf{R})\right|^{2}$. When the dressing laser is far-detuned everywhere so that $\Omega \ll \Delta+V_{\mathrm{URM}}(\mathbf{R})$, the $\mathrm{BO}$ curve becomes $\mathcal{E}(\mathbf{R}) \approx-\Omega^{2} / 2\left[\Delta+V_{\mathrm{URM}}(\mathbf{R})\right]$. If the detuning also satisfies $V_{\mathrm{URM}}(\mathbf{R}) \ll \Delta$, we recover the mixing probability $b(\mathbf{R}) \rightarrow$ $b=|\Omega|^{2} / 2 \Delta^{2}$ from the previous perturbative treatment, and the $\mathrm{BO}$ curve is further simplified to $\mathcal{E}(\mathbf{R}) \approx b V_{\mathrm{URM}}(\mathbf{R})-$ $b \Delta \equiv U(\mathbf{R})-b \Delta$, the perturbative result with a mere shift of the threshold. We note that we select $\Omega$ and $\Delta$ such that the average mixing parameter $\bar{b}$ (defined below) is smaller than $10 \%$; in this regime, the perturbative treatment agrees well with the two-channel model.

\section{RESULTS}

We present the results for dressing ground-state atoms first with $n s$, then $n p$, and $n d$ Rydberg states, using the simplified interaction treatment. This is followed by a section where the effect of the extended interaction is investigated. Finally, we explore how polyatomic URdMs could be created and studied.

\section{A. $s$-type URdM}

We first consider dressing a pair of ground-state atoms by a Rydberg $n s$ state. Since the trilobite potential $V_{\text {URM }}(\mathbf{R})=$ $V_{\mathrm{URM}}(R)$ is isotropic and independent of $\hat{\mathbf{R}}$ for $n s$ states, the radial $R$ dependence is sufficient to illustrate the effect of Rydberg dressing. The left panel of Fig. 3 shows $V_{G}(R)$ between a pair of ground-state atoms and the dressing scheme by the Rydberg state with the corresponding potential $V_{\mathrm{URM}}(R)$. As a practical example, we consider a pair of spin-up $5 s_{1 / 2}$ ground-state ${ }^{87} \mathrm{Rb}$ atoms dressed by $30 s_{1 / 2}$ with $m_{j}=1 / 2$, which can be achieved by using two linearly polarized lasers (see Fig. 1) with chosen parameters $\Omega=25 \times 2 \pi \mathrm{MHz}$ and $\Delta=100 \times 2 \pi \mathrm{MHz}$. Diagonalization of Eq. (5) leads to the BO potential $U(R)$ for the dressed-pair ground state depicted in Fig. 3(a). If the mixing parameter $\bar{b}$ is chosen appropriately, this potential can support vibrational bound levels $v$ which are obtained by solving the effective radial Schrödinger equation

$$
\left[-\frac{1}{m} \frac{d^{2}}{d R^{2}}+U(R)\right] \psi_{v}(R)=E_{v} \psi_{v}(R),
$$

where $m$ is the atomic mass. We define the average mixing parameter indicating how much Rydberg character is imprinted onto the ground-state atoms by $\bar{b}=\int\left|\psi_{v}(R)\right|^{2} b(\mathbf{R}) d R$. For our chosen parameters, the binding energy is $\left|E_{v}\right| \approx 1.11 \times$ $2 \pi \mathrm{MHz}$ with the average mixing parameter $\bar{b} \approx 6.89 \%$. We note that a small $\bar{b}$ implies a much longer bound level lifetime $\tau_{v}$ than that of the trilobite molecule $\tau_{\mathrm{URM}}$, with $\tau_{v} \sim \tau_{\mathrm{URM}} / \bar{b}$; in the example above, roughly 15 times longer. Since $\tau_{\text {URM }} \lesssim$ $\tau_{n}$ and the lifetime $\tau_{n}$ of the Rydberg level $n$ can be estimated by $\tau_{n}=\tau_{0} v^{\lambda}$, where $\tau_{0}$ and $\lambda$ are obtained by experimental fit $[67,68]$, we write $\tau_{v} \lesssim \tau_{0} v^{\lambda} / \bar{b}$. For $\mathrm{Rb}$ in $30 s_{1 / 2}$ with $v=n-\delta_{s}$ (quantum defect $\delta_{s} \approx 3.14, \tau_{0}=1.4 \mathrm{~ns}$, and $\lambda=$ $2.99 \simeq 3$ [68]) we have $\tau_{n} \approx 26.2 \mu$ s, so that with $\bar{b} \approx 6.89 \%$, $\tau_{v} \lesssim 0.38 \mathrm{~ms}$. The lifetime $\tau_{v}$ might also be heavily reduced by chemical reactions [69] in alkali-metal atomic gases such as $\mathrm{Rb}$, while such chemical reactions will be absent in $\mathrm{Sr}$.

Figure 3(a) also depicts $\left|\psi_{v}(R)\right|^{2}$ of the molecular bound state; although extended over roughly 400 Bohr radii $\left(a_{0}\right)$, it is still well localized, which favors its detection (see Sec. IV C 

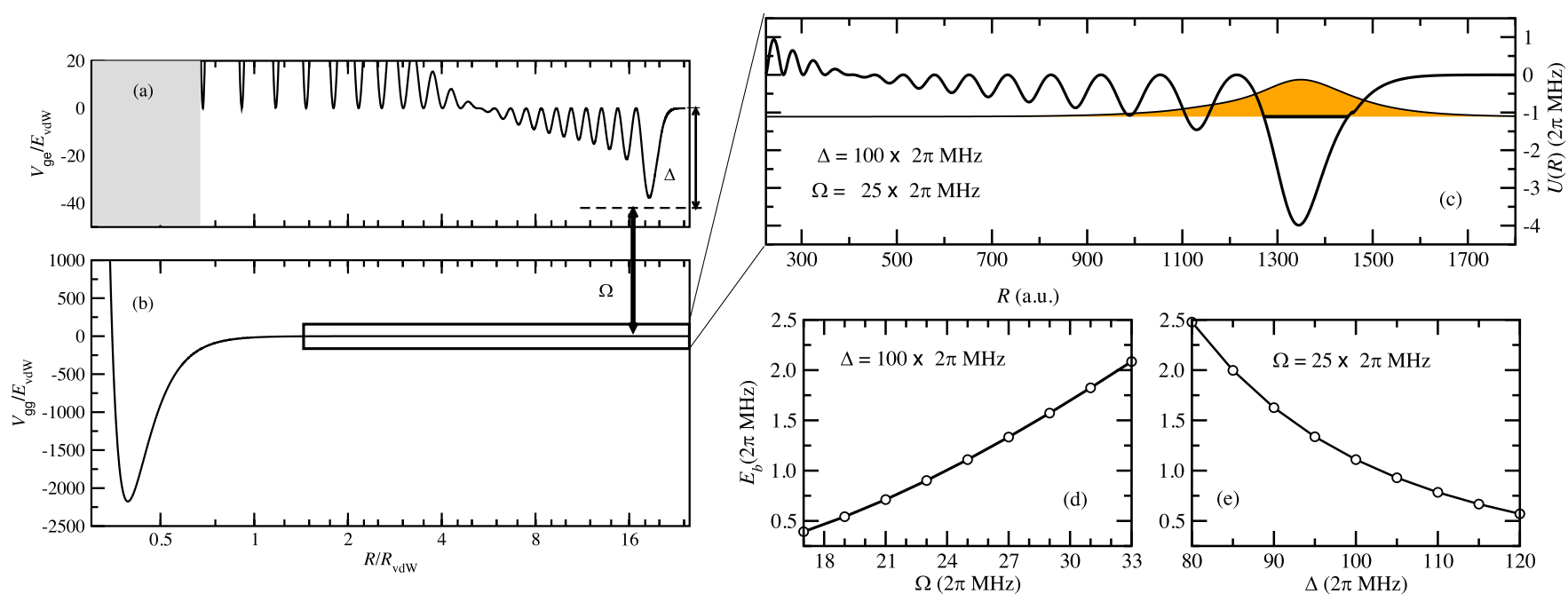

FIG. 3. Left: Sketch of the (a) potentials and (b) dressing lasers. Right: (c) The BO potential $U(R)$ between Rydberg-dressed atoms as a function of interatomic distances $R$. The lowest vibrational level at about $1.11 \times 2 \pi \mathrm{MHz}$ (solid black line) and the corresponding wave function probability (area plot) are also depicted. (d) The binding energy $E_{b}=\left|E_{v=0}\right|$ of the ground state as a function of the Rabi frequency $\Omega$ for a specific detuning $\Delta$. (e) $E_{b}$ as a function of $\Delta$ for a specific value of $\Omega$.

below). In fact, the two atoms are always separated by roughly $1000 a_{0}$, their oscillation never reaching the shorter range where standard chemical bonds take place, as opposed to the very extended bound states realized in Feshbach molecules [4] which span the full range of separations, including short distances.

Rydberg dressing also allows to easily change the mixing parameter $b$ and hence the depth of the BO potential $U(R)$. By either tuning the Rabi frequency $\Omega$ or the detuning $\Delta$ or both, binding energies of the molecular states can be controlled. Binding energies of a few megahertz can be achieved by varying the parameters of the dressing lasers, as shown in Figs. 3(b) and 3(c), where the Rabi frequency $\Omega$ (detuning $\Delta$ ) is changed for a fixed detuning $\Delta$ (Rabi frequency $\Omega$ ), respectively.

\section{B. $p$-type URdM}

As opposed to isotropic $n s$ trilobite potentials, the angular dependence becomes important when dressing with $n p$ or $n d$ states. It manifests itself by the formation of anisotropic potentials which leads to control over the spatial orientation of the dressed ground state (discussed below). We first examine $n p$ states, and focus on dressing with the Rydberg 30p $3 / 2$ state using a one-photon excitation scheme (see Fig. 1) with $\Omega=25 \times 2 \pi \mathrm{MHz}$ and $\Delta=135 \times 2 \pi \mathrm{MHz}$. The left-hand panels of Fig. 4 show the dressed potentials produced by dressing lasers with polarization $\sigma=+1$ [Fig. 4(a)], $\sigma=0$ [Fig. 4(c)], and $\sigma=-1$ [Fig. 4(e)]. The polarization is defined along the $z$ axis, and the cylindrical symmetry about that axis allows for the description of the BO-PES as two-dimensional potential surfaces. We illustrate the BO-PES as a function of $x \equiv R \sin \theta \cos \phi$ and $z$ in Fig. 4, setting $y$ to the specific value $y \equiv R \sin \theta \sin \phi=0$ (i.e., $\phi=0$ ), with the Rydberg ion core sitting at the origin. In Fig. 4(a), $m_{j}=m_{s_{g}}+\sigma=3 / 2$ gives $c_{m_{\ell}}=\delta_{m_{\ell} 1}$ in Eq. (4) and $\Theta=\left|Y_{11}\right|^{2} \propto \sin ^{2}\left(\theta_{R}\right)$, leading to a potential minimum at $\theta_{R}=\pi / 2$ (on the $x$ axis in the $x z$ plane) that can support a localized bound state as shown in Fig. 4(b). The azimuthal symmetry gives a torus about the $z$ axis, sketched between Figs. 4(a) and 4(b), together with the corresponding light polarization in the $x z$ plane. A pair of ground-state atoms can be bound, with one atom at the

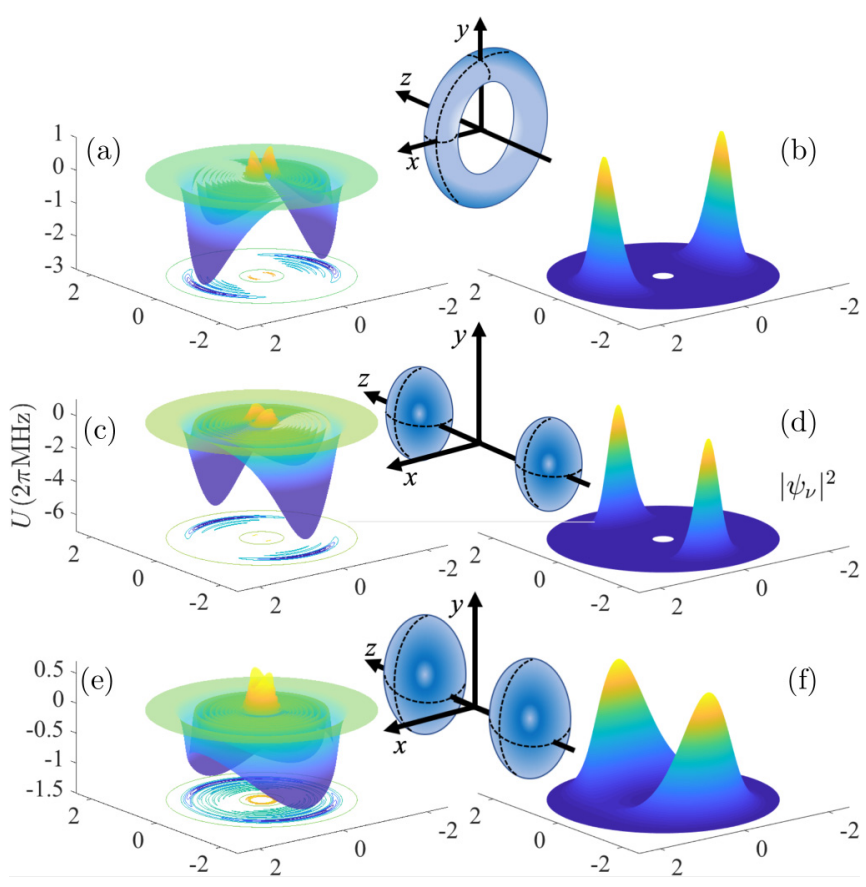

FIG. 4. The Born-Oppenheimer potential surface (with contour projection in the $x z$ plane) and wave functions between two Rydbergdressed $30 p_{3 / 2}{ }^{87} \mathrm{Rb}$ atoms, with $x$ and $z$ axes in units of $1000 a_{0}$.

(a) The potential surface $U(x, y=0, z)$ in units of $2 \pi \mathrm{MHz}$ and (b) the wave function $\left|\psi_{v}(x, y=0, z)\right|^{2}$ for $\sigma=+1$ polarization, (c), (d) for linear polarization $\sigma=0$, and (e), (f) for $\sigma=-1$, respectively. The sketches between the left and right plots depict the wells in space. 


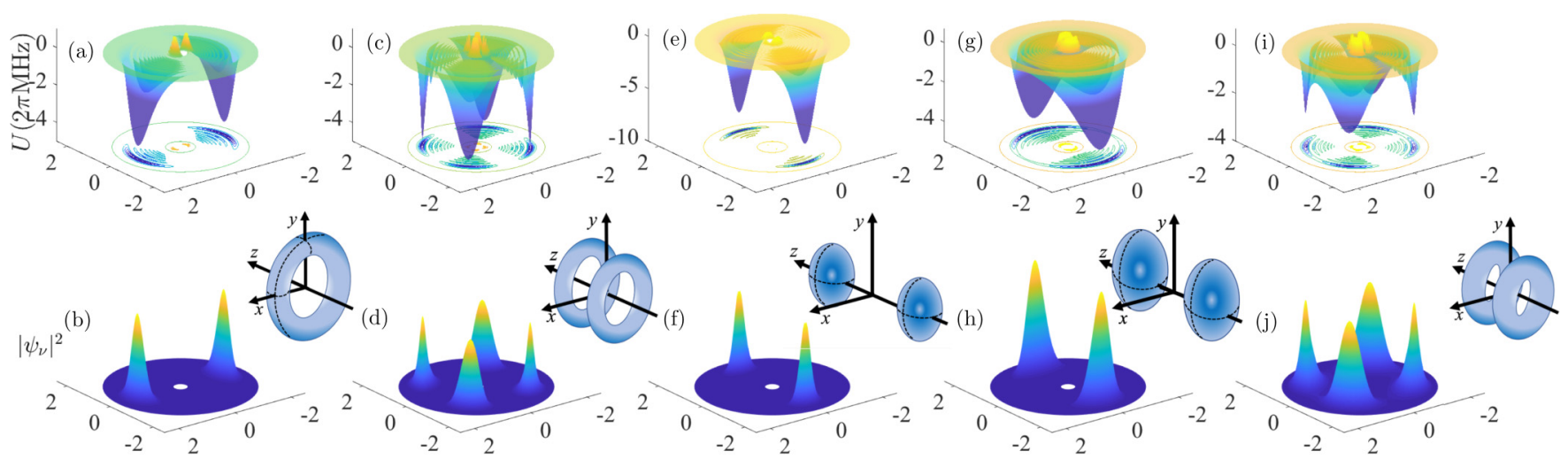

FIG. 5. BO-PES (with contour projection in the $x z$ plane) for $\sigma=2,1,0,-1$, and -2 in (a), (c), (e), (g), and (i), respectively, and corresponding wave functions for Rydberg-dressed $30 d_{5 / 2}{ }^{87} \mathrm{Rb}$ atoms in the $x z$ plane in (b), (d), (f), (h), and (j), where $z$ is the polarization axis. $x$ and $z$ axes are in units of $1000 a_{0}$. The sketches between the top and bottom plots depict the wells in space.

origin and the other in the torus; this molecular state has a binding energy of $\left|E_{v}\right|=0.517 \times 2 \pi \mathrm{MHz}$ and an average mixed parameter $\bar{b}=3.29 \%$. With $\tau_{0}=3.5 \mathrm{~ns}, \lambda=2.90$, and $\delta_{p}=2.64$, we have, for $30 p_{3 / 2}, \tau_{n} \approx 51.49 \mu$ s and $\tau_{v} \lesssim$ $\tau_{n} / \bar{b} \approx 1.6 \mathrm{~ms}$, or roughly 30 times larger.

The linear polarization case with $\sigma=0$ gives $m_{j}=1 / 2$ and leads to two local minima at $\theta_{R}=0$ and $\pi$ shown in Fig. 4(c). These isolated and localized potential wells can support two near-degenerated symmetric and antisymmetric wave functions, though only the symmetric one is permitted for identical bosons such as Rb atoms. Figure 4(d) shows the symmetric state density with $\left|E_{v}\right| \approx 1.10 \times 2 \pi \mathrm{MHz}$ and $\bar{b} \approx 6.30 \%$. The sketch between Figs. 4(c) and 4(d) shows the result due to the azimuthal symmetry about the $z$ axis.

For $\sigma=-1$ with $m_{j}=-1 / 2$, two local minima exist at $\theta_{R}=0$ and $\pi$, as shown in Fig. 4(e); those wells are wider in both $R$ and $\theta_{R}$ when compared to the $\sigma=0$ case. The corresponding bound state shown in Fig. 4(f) has a shallower binding energy $\left|E_{v}\right| \approx 0.136 \times 2 \pi \mathrm{MHz}$ and smaller average mixing $\bar{b} \approx 2.12 \%$. The sketch between Figs. 4(e) and 4(f) shows the result due to the azimuthal symmetry about the $z$ axis.

\section{C. $d$-type URdM}

Finally, using $n d$ dressing states provides additional polarization options. Figure 5 shows the BO-PES in the $x z$ plane fixing $y=0$, with a Rabi frequency $\Omega=25 \times 2 \pi \mathrm{MHz}$ for $\sigma=2,1,0,-1$, and -2 in Figs. 5(a), 5(c), 5(e), 5(g), and 5 (i), and corresponding detunings $\Delta=120 \times 2 \pi, 100 \times 2 \pi$, $150 \times 2 \pi, 100 \times 2 \pi$, and $80 \times 2 \pi \mathrm{MHz}$, respectively. In particular, for $\sigma=1$ and -2 , the potentials have two local minima at $\theta_{R}=\pi / 4$ and $3 \pi / 4$. The azimuthal symmetry leads to a single torus for $\sigma=+2$, double torus for $\sigma=+1$ or -2 , and double oblate spheres for $\sigma=0$ and -1 . We note that for the last two cases $\sigma=-1$ and -2 , the wells are less tight than for the other polarizations. The corresponding molecular wave functions are shown in Figs. 5(b), 5(d), $5(\mathrm{f}), 5(\mathrm{~h})$, and $5(\mathrm{j})$, with binding energies $\left|E_{v}\right| \approx 0.93 \times 2 \pi$, $0.99 \times 2 \pi, 1.34 \times 2 \pi, 0.87 \times 2 \pi$, and $0.50 \times 2 \pi \mathrm{MHz}$ and average mixing parameter $\bar{b} \approx 5.53 \%, 7.07 \%, 7.78 \%, 6.67 \%$, and $6.47 \%$ respectively. The additional polarizations provide more structure for the dressed potentials and could allow more complex polyatomic systems (see below).

\section{Extended model}

In this section, we establish a more sophisticated model including all the spin effects and the singlet and triplet $s$ - and $p$-wave scattering, justifying the validity of our perturbation and two-channel approach. As a concrete example, we study here the case of dressing the hyperfine ground state of ${ }^{87} \mathrm{Rb} \quad\left|g_{\kappa^{*}}\right\rangle=|5 s\rangle\left|F^{\kappa^{*}}=2, m_{F}^{\kappa^{*}}=2\right\rangle=$ $\left|5 s_{1 / 2}, m_{j}^{\kappa^{*}}=1 / 2\right\rangle\left|I^{\kappa^{*}}=3 / 2, m_{I}^{\kappa^{*}}=3 / 2\right\rangle$ with Rydberg states $\quad\left|e_{\eta^{*}}\right\rangle=\left|30 s_{1 / 2}, m_{j}^{\eta^{*}}\right\rangle\left|I^{\eta^{*}}=3 / 2, m_{I}^{\eta^{*}}\right\rangle$ and laser polarization $\sigma=0$. Since $m_{j}^{\eta^{*}}-m_{j}^{\kappa^{*}}=\sigma$ and the dressing laser should not affect the nuclear spin, i.e., $m_{I}^{\eta^{*}}=m_{I}^{\kappa^{*}}$, only one Rydberg state with $m_{j}^{\eta^{*}}=1 / 2$ and $m_{I}^{\eta^{*}}=3 / 2$ is directly coupled. We also focus on systems of two atoms, indicated by $A$ and $B$, in this section. The adiabatic Hamiltonian for the dressed system (at fixed $\mathbf{R}$ ) can be expressed as $\hat{H}(\mathbf{R})=\hat{H}_{1 b}^{(A)}+\hat{H}_{1 b}^{(B)}+\hat{V}_{2 b}(\mathbf{R})$. Here the single-atom Hamiltonian $\hat{H}_{1 b}$ under the rotating-wave approximation is given by

$$
H_{1 b}=\hat{H}_{\mathrm{HF}}+\hat{H}_{\mathrm{Ryd}}+\hat{H}_{P},
$$

where $\hat{H}_{\mathrm{HF}}=A_{\mathrm{HF}} \vec{I} \cdot \vec{S}|5 s\rangle\langle 5 s|$ denotes the hyperfine interaction for a ground-state atom. The hyperfine Hamiltonian is diagonalized in the basis of $\left|g_{\kappa}\right\rangle=|5 s\rangle\left|F^{\kappa} m_{F}^{\kappa}\right\rangle$, where $\left|F^{\kappa} m_{F}^{\kappa}\right\rangle=\sum_{m_{s}^{\kappa}, m_{I}^{\kappa}} C_{s^{\kappa} m_{s}^{\kappa} I^{\kappa}, m_{I}^{\kappa}}^{F^{\kappa} m^{\kappa}}\left|s^{\kappa} m_{s}^{\kappa}\right\rangle\left|I^{\kappa} m_{I}^{\kappa}\right\rangle$. The corresponding eigenenergy is $E_{g}^{\kappa}=\left(A_{\mathrm{HF}} / 2\right)\left[F^{\kappa}\left(F^{\kappa}+1\right)-I^{\kappa}\right.$ $\left.\left(I^{\kappa}+1\right)-s^{\kappa}\left(s^{\kappa}+1\right)\right]$. In contrast, the hyperfine interaction for Rydberg states is negligible, and we include in $\hat{H}_{\text {Ryd }}$ the effects of core electrons and the Rydberg spin-orbit splitting that is typically parametrized by measured quantum defects from atomic spectroscopy. To be specific, $\left|e^{\eta}\right\rangle$ are eigenstates of $\hat{H}_{\mathrm{Ryd}}$, where $\left\langle\mathbf{r}_{e} \mid e_{\eta}\right\rangle=\phi_{n\left(\ell^{\eta} s^{\eta}\right) j^{\eta} m_{j}^{\eta}}\left(\mathbf{r}_{e}\right)\left|I^{\eta} m_{I}^{\eta}\right\rangle$, where $\phi_{n^{\eta}\left(\ell^{\eta} s^{\eta}\right) j^{\eta} m_{j}^{\eta}}\left(\mathbf{r}_{e}\right)=\sum_{m^{\eta}, m_{s}^{\eta}} C_{\ell^{\eta} m^{\eta}, s^{\eta} m_{s}^{\eta}}^{j^{\eta} m_{j}^{\eta}} \mathcal{R}_{v^{\eta} \ell^{\eta}}\left(r_{e}\right) Y_{\ell^{\eta} m^{\eta}}(\hat{r})\left|s^{\eta} m_{s}^{\eta}\right\rangle$ with $v^{\eta}=n^{\eta}-\delta_{n^{\eta} \ell^{\eta} j^{\eta}}$. The corresponding eigenvalues are $E^{\eta}-\bar{E}$, where $E^{\eta}=-0.5 /\left(v^{\eta}\right)^{2}$, and $\bar{E}$ is an energy shift that satisfies $E^{\eta^{*}}-\bar{E}=E_{g}^{\kappa^{*}}+\Delta$ for the target states $\left|g_{\kappa^{*}}\right\rangle$ and $\left|e_{\eta^{*}}\right\rangle$. The coupling term is given by 
$\hat{H}_{P}=(\Omega / 2)\left|5 s_{1 / 2}\right\rangle\left\langle 30 s_{1 / 2}\right|+$ H.c. The two-body trilobite interaction is given by $\hat{V}_{2 b}(\mathbf{R})=\sum_{\mathbf{r}_{e}} \hat{V}_{F}\left(\mathbf{r}_{e}^{A}, \mathbf{R}\right)\left|\mathbf{r}_{e}^{A}\right\rangle_{A}\left\langle\mathbf{r}_{e}^{A}\right| \otimes$ $\left|5 s_{1 / 2}\right\rangle_{B}\left\langle 5 s_{1 / 2}\right|+(A \Leftrightarrow B)$, where $\hat{V}_{F}\left(\mathbf{r}_{e}^{A}, \mathbf{R}\right)$ is given by Eq. (1). In the example here, the semiclassical electronic momentum $k_{R}$ is determined by $n_{H}=27$, the principal quantum number of the nearest hydrogenic manifold to the $30 s_{1 / 2}$ Rydberg state. The quantum defects $\delta_{n \ell j}$, electron-atom scattering phase shift $\eta_{L S}^{J}$, and hyperfine strength $A_{\mathrm{HF}}$ are all taken from Ref. [58].

Due to the Rydberg blockade mechanism, we can neglect double excitations, and expand the Hamiltonians with basis of both atoms in the ground state $\left|\alpha_{\kappa \tau}\right\rangle=$ $\left(\left|g_{\kappa}\right\rangle_{A}\left|g_{\tau}\right\rangle_{B}+\left|g_{\tau}\right\rangle_{A}\left|g_{\kappa}\right\rangle_{B}\right) / \sqrt{2\left(1+\delta_{\kappa \tau}\right)}$ or one atom in the ground state and the other one in an excited state $\left|\beta_{\kappa \eta}\right\rangle=$ $\left(\left|g_{\kappa}\right\rangle_{A}\left|e_{\eta}\right\rangle_{B}+\left|e_{\eta}\right\rangle_{A}\left|g_{\kappa}\right\rangle_{B}\right) / \sqrt{2}$. Numerically, we restrict our basis set to the states energetically close to $n_{H}=25-28$. We can further reduce the size of the basis due to the spherical symmetry of $s$ states. Under this spherical symmetry, the direction of quantization is no longer relevant, and we choose the molecular axis to be the $z$ axis for convenience. The spherical symmetry of the system also ensures the total hyperfine projection $m_{F_{A}}+m_{F_{B}}$ is a good quantum number, where the quantum number of hyperfine spin and its projection of atom $A(B)$ are denoted as $F_{A}\left(F_{B}\right)$ and $m_{F_{A}}\left(m_{F_{B}}\right)$. This restriction allows only one $g+g$ basis $\left|\alpha^{*}\right\rangle \equiv\left|\alpha_{\kappa^{*} \kappa^{*}}\right\rangle$ with total hyperfine projection equal to $m_{F_{A}}+m_{F_{B}}=4$ in our example. For the $g+e$ basis $\left|\beta_{\kappa \eta}\right\rangle$, the good quantum number of the combined system is $m_{\Omega}=m_{s}^{\kappa}+m_{I}^{\kappa}+m_{j}^{\eta}$, which should be equal to the ground state $m_{\Omega}=m_{F_{A}}+m_{F_{B}}-m_{I}^{\kappa^{*}}$. The dressing laser does not affect the nuclear spin, $m_{I}^{\eta}=m_{I}^{\kappa^{*}}=3 / 2$, which leads to $m_{\Omega}=5 / 2$. For convenience, we also denote the special basis that can be directly coupled to the ground state as $\left|\beta^{*}\right\rangle \equiv$ $\left|\beta_{\kappa^{*} \eta^{*}}\right\rangle$.

Using this two-atom basis, the Hamiltonian for the dressed system can be expressed as

$$
H(R)=\left[\begin{array}{ll}
H_{g g} & H_{g e} \\
H_{e g} & H_{e e}
\end{array}\right],
$$

where $H_{g g}=E_{\alpha^{*}}\left|\alpha^{*}\right\rangle\left\langle\alpha^{*}\right|$ is the Hamiltonian for two groundstate atoms with $E_{\alpha^{*}}=E_{g}^{\kappa^{*}}+E_{g}^{\kappa^{*}} . H_{e e}$ is the same trilobite Hamiltonian of a Rydberg atom and a ground-state atom perturber as in Ref. [58] (with a proper symmetrization and a constant energy shift from the rotating-wave approximation), which can be diagonalized and gives $H_{e e}=\sum_{\gamma} E_{\gamma}|\gamma\rangle\langle\gamma|$. The eigenvalues $E_{\gamma}=U_{\gamma}(R)+E_{g}^{\kappa^{*}}+\Delta$, where $U_{\gamma}(R)$ are the trilobite potentials for $m_{\Omega}=5 / 2$ calculated using the same method as in Ref. [58], are shown in Fig. 6. The corresponding eigenvectors $|\gamma\rangle=\sum_{\beta} \Phi_{\beta}^{(\gamma)}|\beta\rangle$ are obtained from diagonalization and give the form of $H_{g e}$ as

$$
H_{g e}=\sum_{\gamma} \frac{\Omega}{\sqrt{2}} \Phi_{\beta^{*}}^{(\gamma)}\left|\alpha^{*}\right\rangle\langle\gamma|
$$

Therefore, $\left|\Phi_{\beta^{*}}^{(\gamma)}\right|^{2}$ characterizes how strongly a trilobite potential $U_{\gamma}$ is coupled to the ground state, indicated by the color scheme in Fig. 6. One can see that only one trilobite potential curve is strongly coupled to the ground state (in blue), justifying the two-channel approach.

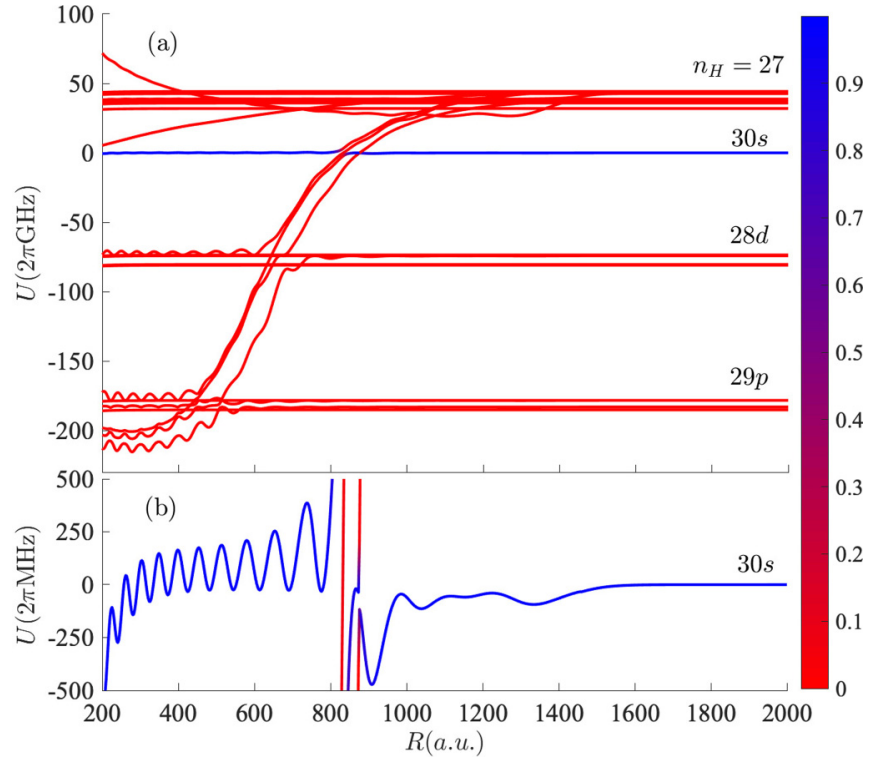

FIG. 6. (a) Trilobite potential curves for ${ }^{87} \mathrm{Rb}$ with $m_{\Omega}=5 / 2$ near the $n_{H}=27$ perturbed hydrogenic manifold. All spin effects and $s$ - and $p$-wave scattering are included. The color indicates the probability of each eigenstate projection onto the $30 s+5 s$ basis, determining the Rydberg-dressing coupling strength to the two-body ground-state $5 s+5 s$ channel. (b) Zoom of the potential curves near the $5 s+30 s$ threshold.

Nevertheless, we diagonalize the Hamiltonian in Eq. (8) with all channels and obtain BO potentials, shown in Fig. 7 for the parameter $\Omega=20 \times 2 \pi \mathrm{MHz}$ and $\Delta=120 \times 2 \pi \mathrm{MHz}$. Although including the $p$ wave can significantly affect the shape of potential, it is still deep enough to support localized molecular state. Here, to ensure the validity of the adiabatic approximation, we directly solve the full coupled equations for the bound state, i.e.,

$$
\left[\begin{array}{cc}
-\frac{1}{m} \frac{d^{2}}{d R^{2}}+H_{g g} & H_{e g} \\
H_{g e} & -\frac{1}{m} \frac{d^{2}}{d R^{2}} I+H_{e e}
\end{array}\right]\left[\begin{array}{l}
\Psi_{g} \\
\Psi_{e}
\end{array}\right]=E_{v}\left[\begin{array}{l}
\Psi_{g} \\
\Psi_{e}
\end{array}\right] .
$$

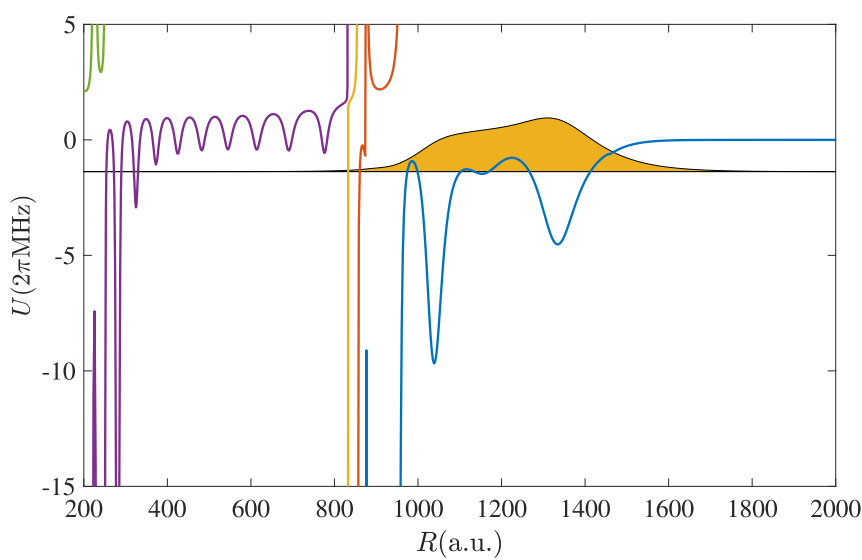

FIG. 7. The BO potentials $U$ between Rydberg-dressed atoms as a function of interatomic distance $R$. The ground-state atoms are dressed by the trilobite potentials shown in Fig. 6. A vibrational level at about $1.37 \times 2 \pi \mathrm{MHz}$ (solid black line) and the corresponding wave function probability (area plot) are also depicted. 
The area plot in Fig. 7 shows the wave function $\left|\Psi_{g}(R)\right|^{2}$ with the base line indicating the bound-state energy at $\left|E_{v}\right|=$ $1.37 \times 2 \pi \mathrm{MHz}$. The averaged mixing can now be given by $1-\int d R\left|\Psi_{g}(R)\right|^{2} \approx 6.65 \%$. Although the details of the URdM potential, bound level, and wave function are different from the simpler treatment of the previous sections, the qualitative picture remains valid. In fact, we obtain a small averaged mixing parameter $\bar{b}$ of roughly 7\%, a binding energy of the same order as before, and a well-localized bound state, confirming the validity of the results of the simpler treatment.

\section{E. Polyatomic molecules}

In previous sections, we showed that by choosing particular laser polarizations, e.g., dressing $n p_{3 / 2}$ states with $\sigma=0$ as shown Figs. 4(c) and 4(d), the corresponding BO-PES can have separated local minima. Hence, a trimer state can exist, with each local minimum binding one ground-state atom to the central Rydberg atom. As in the diatomic case, the interaction $V_{G}$ between the two, three, or more groundstate atoms can be neglected. Moreover, by using additional dressing lasers, more complex PESs can be engineered with more local minima for polyatomic molecules.

We illustrate the concept within the perturbation treatment, by considering multiple lasers characterized by $\Omega_{k}, \Delta_{k}$, and $\sigma_{k}$ dressing to different Rydberg states $\left|e^{(k)}\right\rangle$. We also focus on the case where the spatial overlapping between these selected Rydberg states can be neglected, i.e., $\left\langle e^{(k)} \mid \mathbf{r}_{e}\right\rangle\left\langle\mathbf{r}_{e} \mid e^{\left(k^{\prime}\right)}\right\rangle \approx 0$, which can be fulfilled by selecting Rydberg states with distinct $n$ and $m_{j}$. Neglecting the trilobite interaction (zero order), the collective ground state of $N$ Rydberg-dressed identical atoms within one Rydberg blockade domain can be written as $\left|G_{N}^{*}\right\rangle=\alpha_{N}\left|G_{N}\right\rangle+\sum_{k} \beta_{N}^{(k)}\left|S_{N}^{(k)}\right\rangle$, where $\left|G_{N}\right\rangle=$ $\left|g_{1}, g_{2}, \ldots, g_{N}\right\rangle$ and $\left|S_{N}^{(k)}\right\rangle=\frac{1}{\sqrt{N}} \sum_{i=1}^{N}\left|E_{i}^{(k)}\right\rangle$ with $\left|E_{i}^{(k)}\right\rangle$ as a shorthand notation for $\left|g_{1}, g_{2}, \ldots, e_{i}^{(k)}, \ldots, g_{N}\right\rangle$. When all the detunings are large enough that $\Omega_{k} \ll \Delta_{k}$, we have $\beta_{N}^{(k)}=\sqrt{N} \Omega_{k} / 2 \Delta_{k}$, where the factor $\sqrt{N}$ indicates a collective enhancement, which gives the mixing parameter $b_{N}^{(k)} \equiv\left|\beta_{N}^{(k)}\right|^{2}=N\left|\Omega_{k}\right|^{2} / 4 \Delta_{k}^{2}$. For Rydberg states with low angular momentum $(\ell \leqslant 2)$, the trilobite interaction can be written as $\hat{V}_{\text {tot }}=\sum_{k, i, j \neq i} V_{\mathrm{URM}}^{(k)}\left(\mathbf{R}_{i j}\right)\left|E_{i}^{(k)}\right\rangle\left\langle E_{i}^{(k)}\right|$, where the couplings between different Rydberg states are neglected due to their negligible spatial overlap. In the spirit of Born-Oppenheimer approximation, for a fixed set of $\left\{\mathbf{R}_{i j}\right\}$, the adiabatic potential surface is therefore given by $\left\langle G_{N}^{*}\left|\hat{V}_{\text {tot }}\right| G_{N}^{*}\right\rangle=\frac{1}{N} \sum_{k} b_{N}^{(k)} \sum_{i, j \neq i} V_{\mathrm{URM}}^{(k)}\left(\mathbf{R}_{i j}\right)=$ $\sum_{k, i, j<i} b_{k} V_{\mathrm{URM}}^{(k)}\left(\mathbf{R}_{i j}\right)$, where $b_{k} \equiv\left|\Omega_{k}\right|^{2} / 2 \Delta_{k}^{2}$ is the two-body mixing parameter for the $k$ th laser. Therefore, the polyatomic BO-PES can be regarded as a summation of the two-body single-laser BO-PES, so that the corresponding binding energies can be roughly given by the summation of the dimer binding energies as long as the local minima are well separated.

Figure 8 shows the two-body BO-PES for a two-color dressing scheme, with one laser dressing the ground state with the $30 p_{3 / 2}$ Rydberg state $\left(\Delta_{1}=135 \times 2 \pi \mathrm{MHz}, \Omega_{1}=\right.$ $25 \times 2 \pi \mathrm{MHz}$, and $\left.\sigma_{1}=0\right)$, and a second laser dressing with $35 p_{3 / 2}\left(\Delta_{2}=50 \times 2 \pi \mathrm{MHz}, \Omega_{2}=15 \times 2 \pi \mathrm{MHz}\right.$, and

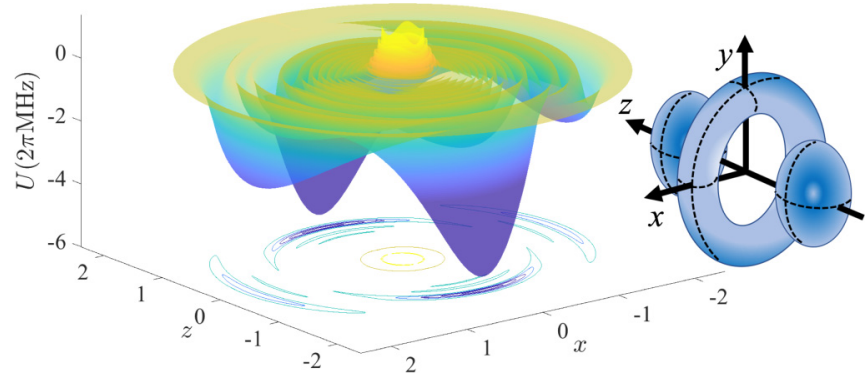

FIG. 8. Two-body BO-PES for Rydberg-dressed atoms with twocolor dressing scheme. One $\sigma_{1}=0$ laser dresses the $5 s_{1 / 2}$ groundstate atom with the $30 p_{3 / 2}$ state, while the other laser $\sigma_{2}=+1$ dresses to the $35 p_{3 / 2}$ state. The BO-PES shows multiple local minima, implying that polyatomic states with corresponding geometry can be supported.

$\left.\sigma_{2}=+1\right)$. The potential exhibits two minima at $R \approx 1400 a_{0}$ and $\theta_{R}=0$ and $\pi$, respectively, and another local minimum at $R \approx 2000 a_{0}$ and $\theta_{R} \approx \pi / 2$. Therefore, this configuration could sustain a tetramer state, with one atom (Rydberg core) in the center, one in the torus, and one in each sphere. More complex structures can be engineered by selecting different dressing states or laser parameters (e.g., $\sigma$ ) and/or additional dressing states.

\section{DISCUSSION}

In this section, we first elaborate on the difference between the URdMs obtained by the proposed Rydberg dressing, and the OFRs using URM states recently explored. We then discuss the scaling of our results with levels $n$, to finally describe detection schemes.

\section{A. Difference between URdM and OFR}

Recently, OFR based on coupling two scattering groundstate atoms to a URM state via lasers has been explored both theoretically [56] and experimentally [57]. When the detuning is relative large (i.e., off resonance), the real part of the scattering length can be tuned to some large value while the decay remains small. Although that URM-OFR scheme seemingly shares similarities with our URdM proposal, there are several subtle but important differences that are worth discussing here.

Comparing with the OFR studies, the detuning in our scheme is much larger. While this seems only to be a slight technical difference, the resulting mechanism is actually very different. In OFR studies, the detuning from a URM state can be either red or blue and is usually smaller than the zero-point energy near the local minimum that supports the corresponding URM state. Therefore, the laser-dressed $g+g$ threshold can cross the URM potential near that local minimum. As a consequence, a Feshbach molecule formed by this OFR mechanism would strongly couple with a URM state and be dominated by the Rydberg component. In contrast, the detuning from the URM potential threshold in our URdM scheme is always red and larger than the local minimum that supports the URM states. For such large detuning (and consequently large Rabi coupling frequency), we expect that the coupling 
to other URM states can no longer be neglected, and a formal treatment that considers the full potential calculation as our approach is necessary. As a consequence, our potential well is weakly coupled to the URM potential near the minimum locally, and hence the URdM states have a small Rydberg component even when it is tuned near the threshold. This implies that URdM states have a much longer lifetime than the Feshbach molecule states in the OFR scheme for the same $\Omega / \Delta$. From the scattering perspective, the OFR is a closed-channel dominated resonance, while the corresponding scattering resonance associated with URdM states is an openchannel dominated potential resonance.

The physical picture here is that the ground-ground interaction Born-Oppenheimer potentials are modified by mixing in some of the URM character, but the URM states supported by those URM potentials are, to a large extent, irrelevant. This point becomes more obvious for the $p$ - and $d$-wave URdM potentials and states. Notice that the $p$ - and $d$-wave URM potentials are spherically symmetric, while the $p$ - and $d$-wave URdM potentials are anisotropic, since the coupling laser breaks spherical symmetry. The corresponding URdM-state wave functions are therefore very different than those of URM states. The spatial orientation of URdM states, as a result, can be controlled by the laser polarization. The polyatomic URdMs studied here are also completely different than the polyatomic URMs bound by mainly a single Rydberg state, where the binding mechanism of the former is based on laser coupling to different Rydberg states (of different Rydberg manifolds).

\section{B. Variation with level $n$}

By selecting Rydberg-dressing states with various $n$, we can take advantage of the rapid scaling of the position, spatial extent, and lifetime of the trilobitelike states. For simplicity and to avoid the influence of the precise choice of $\Delta$ and $\Omega$, we focus on cases where $\Delta$ is large enough so that the perturbation treatment is appropriate, and choose a fixed mixing parameter $\Omega^{2} / 2 \Delta^{2}=8 \%$. Figure 9 shows the dependence of binding energy $E_{b}$ on the effective principle quantum number $v=n-\delta_{n \ell j}$, with Fig. 9(a) depicting the $\sigma=0$ cases for $n \ell_{j}$ states. For a given $\ell$ value, the $\sigma=0$ dressing gives deeper bound states than the other polarizations, as the corresponding potential wells are sharper and deeper (see Figs. 4 and 5). These $\sigma=0$ polarization wells support levels bound by (15) $\times 2 \pi \mathrm{MHz}$ for $v \sim 30$. Figures 9(b) and 9(c) show the $\sigma$ dependence for $n p_{3 / 2}$ and $n d_{5 / 2}$ states, respectively; in both cases, $\sigma=0$ gives more deeply bound levels. Similar results (not shown here) are obtained for $n p_{1 / 2}$ and $n d_{3 / 2}$ states.

As shown in Fig. 9, the binding energy scales roughly as $v^{-6}$. This can be understood via Eq. (3), as the dressed well is proportional to $V_{\mathrm{URM}} \propto \mathcal{R}_{v \ell}^{2}$. Since $\mathcal{R}_{v \ell}=W_{\nu \ell}(R) / R \propto$ $C_{\nu \ell} R^{-3 / 4}$, where $C_{v \ell} \propto v^{-3 / 2}$ is a constant [62], then $\mathcal{R}_{\nu \ell}^{2} \propto$ $v^{-3} R^{-3 / 2}$, where $R \sim v^{2}$ for the outer wells. As a result, $V_{\mathrm{URM}} \propto v^{-6}$ and so is $E_{b}$. This can also be understood noting that the trilobite wells scale as the electronic density, itself scaling as the inverse of the Rydberg volume or $R^{-3} \propto v^{-6}$. Other details, such as how the scattering length $a_{s}^{T}$ depends on $v$, explain the slight deviation from the $v^{-6}$ scaling.

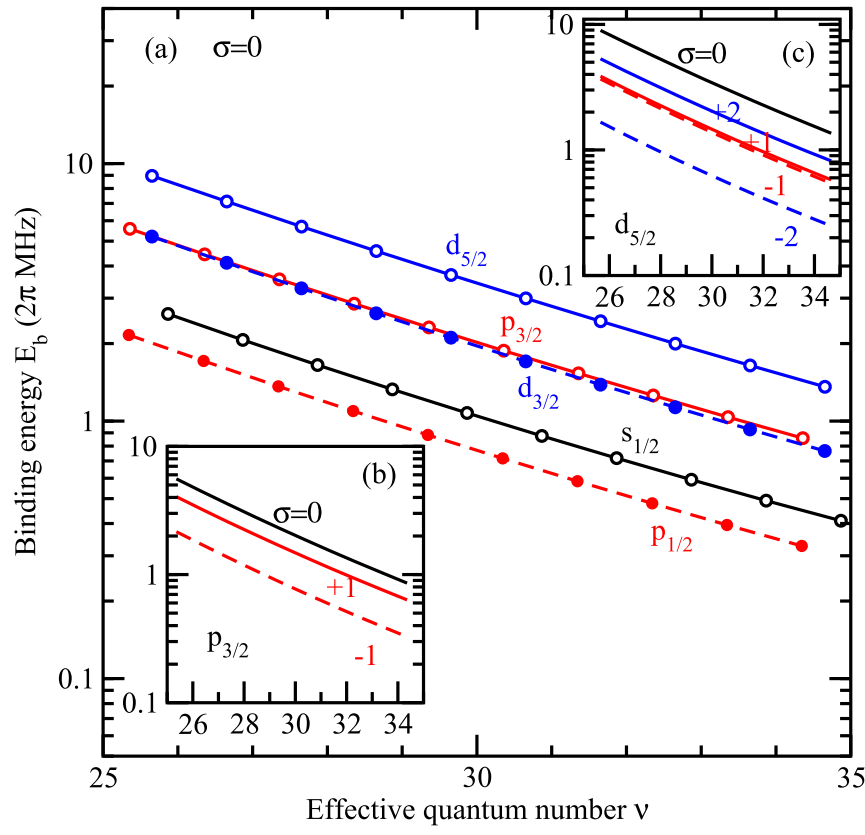

FIG. 9. The binding energy $E_{b}$ for Rydberg dressing with different principle quantum number $n$ with a fixed mixing parameter $\Omega^{2} / 2 \Delta^{2}=8 \%$ in the perturbation treatment: (a) $\sigma=0$ only for $n \ell_{j}$; (b), (c) results for different polarizations $\sigma$ for $n p_{3 / 2}$ and $n d_{5 / 2}$, respectively.

\section{Detection schemes}

A variety of schemes could be employed to detect the new ultralong-range dressed ground-state molecules. We describe two straightforward approaches relying on spectroscopic measurement of atomic hyperfine population, using two-step and state-selective detection schemes. Figure 10 sketches the detection scheme for one of them: as before, we focus on ${ }^{87} \mathrm{Rb}$ as a specific example, which has hyperfine ground states $\left|F, m_{F}\right\rangle$ labeled by the quantum number $F=1$ and 2 and its projection $m_{F}$ onto the quantization axis, where $\mathbf{F}=\mathbf{i}+\mathbf{s}$ is the total spin with $\mathbf{i}$ and $\mathbf{s}$ being the nuclear and electronic spin, respectively.

As depicted in Fig. 10(a), we consider two hyperfine ground states that can be rf coupled, such as $\left|g_{1}\right\rangle \equiv|1,1\rangle$ and $\left|g_{2}\right\rangle \equiv|2,2\rangle$. Other hyperfine states could be considered; however, $|2,2\rangle$ provides a cycling transition with the $5 p$ excited hyperfine state $|3,3\rangle$, thus fixing $|1,1\rangle$ as the other hyperfine ground states. A pair of ground-state atoms will have three energies $E_{1+1}, E_{1+2}$, and $E_{2+2}$ corresponding to both atoms in $\left|g_{1}\right\rangle$, one in $\left|g_{1}\right\rangle$ and one in $\left|g_{2}\right\rangle$, and both in $\left|g_{2}\right\rangle$, respectively. The large hyperfine splitting between the $F=1$ and 2 ground states of ${ }^{87} \mathrm{Rb}$ atoms $\Delta E_{\mathrm{hf}}=h \nu_{\mathrm{hf}}=6.83 \mathrm{GHz}$ [70] allows to dress a particular pair of ground-state atoms without greatly affecting other combinations of hyperfine states of ground-state atoms. By selecting the parameters $\Delta$ and $\Omega$ of the dressing laser carefully, a specific dressed pair $|g g\rangle$ will support molecular bound levels, while the other combinations will be too shallow. Figure 10(a) shows a red-detuned laser from a Rydberg state $|e\rangle$, e.g., $|n s\rangle$, with $\Delta$ such that $\left|g_{2}\right\rangle$ has the strongest coupling to $|e\rangle$. Figure 10(b) depicts how the trilobitelike state correlated to $g+e$ will dress the ground-state pairs with different strengths, with 
(a) single atom: dressing $g_{2}$

(b) atom pair: dressing $g_{2}$
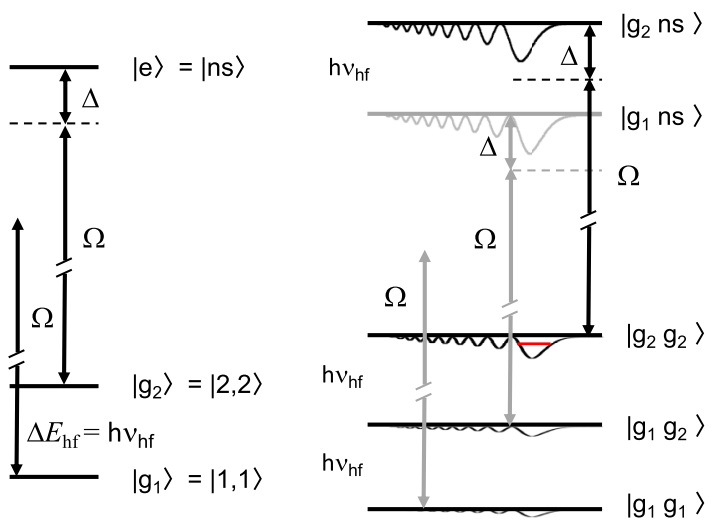

(c) rf-spectroscopy for $g_{2}$

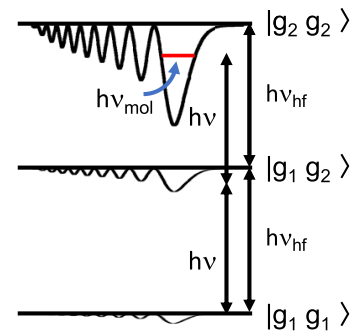

(d) signal for $\mathrm{g}_{2}$

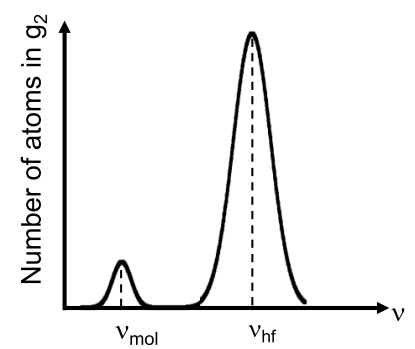

FIG. 10. Detection scheme. (a) Single-atom picture: hyperfine ground states $\left|g_{1}\right\rangle \equiv\left|F=1 m_{F}=1\right\rangle$ and $\left|g_{2}\right\rangle \equiv|2,2\rangle$ dressed by the Rydberg state $|e\rangle=|n s\rangle$ using a laser of Rabi frequency $\Omega$. The detuning $\Delta$ for $\left|g_{2}\right\rangle$ is much smaller than the detuning $\Delta+\Delta E_{\mathrm{hf}}$ for $\left|g_{1}\right\rangle$. (b) Atom-pair picture: the trilobitelike state corresponding to $|g, e\rangle$ is coupled to the three ground-state combinations, with the strongest coupling corresponding to $\left|g_{2} g_{2}\right\rangle$ supporting a bound level, while the weaker coupling for $\left|g_{1} g_{2}\right\rangle$ and $\left|g_{1} g_{1}\right\rangle$ does not sustain a bound level. (c) rf spectroscopy of the dressed-bound level: sample prepared purely in $\left|g_{1}\right\rangle$ is swept by rf photon of frequency $v$ red-detuned from the $\left|g_{2}\right\rangle$, populating the bound state at $v=v_{\mathrm{mol}}$. (d) Corresponding signal showing a small molecular feature reddetuned from the atomic line at $v_{0}$.

$g_{2}+g_{2}$ having the strongest coupling, $g_{1}+g_{2}$ a weaker coupling, and $g_{1}+g_{1}$ a much smaller coupling, due to larger detuning $\Delta+h \nu_{\mathrm{hf}}$ of $g_{1}$. Correspondingly, the pair $g_{2}+g_{2}$ has a long-range well deep enough to support a bound state, while the other combinations $g_{1}+g_{2}$ and $g_{1}+g_{1}$ do not support bound states.

In an atomic sample initially prepared in $\left|g_{1}\right\rangle=|1,1\rangle$, a rf photon can transfer population into the higher hyperfine state $\left|g_{2}\right\rangle=|2,2\rangle$ as shown in Fig. 10(c). By scanning the frequency $v$ of the $\mathrm{rf}$ radiation, the dressed molecular bound level will be populated and a red-detuned signal from the atomic line $v_{\mathrm{hf}}$ corresponding to the bound level $v_{\text {mol }}$ should appear as depicted in Fig. 10(d). The cycling transition between $\left|g_{2}\right\rangle=|2,2\rangle$ and the atomic excited $5 p$ hyperfine state $|3,3\rangle$ can be used to detect the population in $\left|g_{2}\right\rangle$ via atomic fluorescence of the sample. We note again that different sets of hyperfine states could be employed that would affect the detection scheme, e.g., by using ionization of ground state levels instead of optical cycling.

The second approach is based on Coulomb explosion. After creating a dressed molecular ultralong-range ground level as described above, one could ionize both atoms of a pair which would then fly apart due to Coulomb repulsion. Similar to the observation of macrodimers [42] using grids to detect the ions with both temporal and spatial resolution, information about the atoms' separation in the bound state could be gained from correlation in the time of flight, in addition to the number of pairs from the integrated signal.

These two-step (separated-in-time) detection schemes rely on rf spectroscopy to populate the bound level, and stateselective detection. Besides using the optical $5 p$ cycling transition, one could also use the narrower $6 p$ state, or an intermediate Rydberg state followed by field ionization. Finally, one could always use a nearby URM populated level and let it decay into the URdM as a means to produce them.

\section{SUMMARY AND CONCLUSIONS}

We have explored the possibility of imprinting Rydberg character to ground-state atoms using lasers detuned from highly excited Rydberg states. In particular, we showed that Rydberg trilobitelike potentials could be mapped onto the interaction potential of a pair of ground-state atoms, leading to ultralong-range wells. By selecting appropriate laser parameters, we predicted that those wells could sustain bound levels, giving rise to a new type of ultralong-range molecular state. Indeed, as opposed to extended Feshbach molecules for which the bound state can cover a wide range of separations, this new binding mechanism provides localized levels at very large separation. By varying the laser parameters (including their polarizations) and the dressing Rydberg level ( $n s, n p$, or $n d$ ), one can control not only the binding energy and lifetime of these molecules, but also their geometry and orientation. In fact, we showed that $n p$ or $n d$ states can give multiple wells in specific orientation with respect to the laser polarization. This furthermore provides multiple wells to create ultralong-range polyatomic molecules. In addition, by considering multiple Rydberg dressing states, more complex long-range structures can be engineered.

We described detection schemes based on two-step approaches, relying first on rf spectroscopy to populate the bound level followed by a state-selective detection. The signature of the molecular state will be a feature red-detuned from the atomic line, with its magnitude proportional to the number of pairs of ground-state atoms located at the proper separation corresponding to the location of the dressed longrange well. The probability of having a pair of distinguishable atoms separated by a distance $r$ in a homogeneous gas of density $\rho$ is given by $P(r) d r=4 \pi \rho r^{2} e^{-\frac{4}{3} \pi \rho r^{3}} d r$ [71], and for $\rho \approx 10^{12} \mathrm{~cm}^{-3}$, the well corresponding to $30 \mathrm{~s}$ (see Fig. 3 ) located at $r \approx 1350 a_{0}$ and spread over $\Delta r \approx 100 a_{0}$ gives roughly $P(r) \Delta r \approx 3 \times 10^{-4}$. For a small sample containing 10000 atoms, roughly three pairs could be formed. Naturally, increasing the density would lead to more pairs and a stronger signal; e.g., with $\rho \approx 10^{13} \mathrm{~cm}^{-3}, P(r) \Delta r \approx 0.003$ and roughly 30 pairs could be formed, while $\rho \approx 10^{14} \mathrm{~cm}^{-3}$ would lead to $P(r) \Delta r \approx 0.03$ and roughly 300 pairs (however, at such high $\rho$, the density shift would make the experiment challenging). 
We note that one could use Feshbach resonances to increase the probability of pairs at shorter separations near the trilobitelike well. While larger densities lead to larger signal, the particle statistics afforded by quantum degenerate atomic gases would also affect the results. As recently reported in Ref. [72], trilobitelike states can probe two-body correlation functions in degenerate Fermi or Bose gases as well as for distinguishable particles. Additional information about anisotropy in interactions or distributions could potentially be probed by the spatial dependence of the dressed ultralong-range molecular states like $n p$ or $n d$, or polyatomic cases.

Finally, by producing bound pairs or even large polyatomic molecules by Rydberg-dressing ground-state interactions, one could also engineer correlation functions in the gas. As the dressing lasers are turned off, the new distribution would retain the signature of the dressed molecules, and hence could be used to design particular two-body, three-body, or more correlation functions, and even create real three-, four-, or more-body interactions [73]. Overall, Rydberg-dressed systems would enable further studies of many-body phenomena in ultracold gases, in addition to the investigation of peculiar chemical bonds.

\section{ACKNOWLEDGMENTS}

We thank Matthew T. Eiles and Chris H. Greene for helpful discussions and providing $e-\mathrm{Rb}$ scattering phase shifts used in calculating trilobite potentials in our extended model, as well as Jovica Stanojevic for helpful discussions. This work is partially supported by the Australian Research Council (ARC) Discovery Programs, Grants No. DE180100592 and No. DP190100815 (J.W.), and by the National Science Foundation Grant No. PHY-1806653 (R.C.).
[1] M. H. Anderson, J. R. Ensher, M. R. Matthews, C. E. Wieman, and E. A. Cornell, Observation of Bose-Einstein condensation in a dilute atomic vapor, Science 269, 198 (1995).

[2] C. C. Bradley, C. A. Sackett, J. J. Tollett, and R. G. Hulet, Evidence of Bose-Einstein Condensation in an Atomic Gas with Attractive Interactions, Phys. Rev. Lett. 75, 1687 (1995).

[3] K. B. Davis, M. O. Mewes, M. R. Andrews, N. J. van Druten, D. S. Durfee, D. M. Kurn, and W. Ketterle, Bose-Einstein Condensation in a Gas of Sodium Atoms, Phys. Rev. Lett. 75, 3969 (1995).

[4] C. Chin, R. Grimm, P. Julenne, and E. Tiesinga, Feshbach resonances in ultracold gas, Rev. Mod. Phys. 82, 1225 (2010).

[5] Z. Pavlović, R. V. Krems, R. Côté, and H. R. Sadeghpour, Magnetic Feshbach resonances and Zeeman relaxation in bosonic chromium gas with anisotropic interaction, Phys. Rev. A 71, 061402(R) (2005).

[6] M. Gacesa, P. Pellegrini, and R. Côté, Feshbach resonances in ultracold ${ }^{6,7} \mathrm{Li}+{ }^{23} \mathrm{Na}$ atomic mixtures, Phys. Rev. A 78, 010701(R) (2008).

[7] M. Greiner, C. A. Regal, and D. S. Jin, Emergence of a molecular Bose-Einstein condensate from a Fermi gas, Nature (London) 426, 537 (2003).

[8] S. Jochim, M. Bartenstein, A. Altmeyer, G. Hendl, S. Riedl, C. Chin, J. H. Denschlag, and R. Grimm, Bose-Einstein condensation of molecules, Science 302, 2101 (2003).

[9] M. W. Zwierlein, J. R. Abo-Shaeer, A. Schirotzek, C. H. Schunck, and W. Ketterle, Vortices and superfluidity in a strongly interacting Fermi gas, Nature (London) 435, 1047 (2005).

[10] T. Yefsah, A. T. Sommer, M. J. H. Ku, L. W. Cheuk, W. Ji, W. S. Bakr, and M. W. Zwierlein, Heavy solitons in a fermionic superfluid, Nature (London) 499, 426 (2013).

[11] M. J. H. Ku, W. Ji, B. Mukherjee, E. Guardado-Sanchez, L. W. Cheuk, T. Yefsah, and M. W. Zwierlein, Motion of a Solitonic Vortex in the BEC-BCS Crossover, Phys. Rev. Lett. 113, 065301 (2014)

[12] T. Kraemer, M. Mark, P. Waldburger, J. G. Danzl, C. Chin, B. Engeser, A. D. Lange, K. Pilch, A. Jaakkola, H.-C. Nägerl, and
R. Grimm, Evidence for Efimov quantum states in an ultracold gas of caesium atoms, Nature (London) 440, 315 (2006).

[13] M. Berninger, A. Zenesini, B. Huang, W. Harm, H.-C. Nägerl, F. Ferlaino, R. Grimm, P. S. Julienne, and J. M. Hutson, Universality of the Three-Body Parameter for Efimov States in Ultracold Cesium, Phys. Rev. Lett. 107, 120401 (2011).

[14] J. Wang, J. P. D'Incao, B. D. Esry, and C. H. Greene, Origin of the Three-Body Parameter Universality in Efimov Physics, Phys. Rev. Lett. 108, 263001 (2012).

[15] D. Shu, I. Simbotin, and R. Côté, Threshold behavior for the Efimov effect, Chem. Phys. Chem. 17, 3655 (2016).

[16] I. Bloch, Ultracold quantum gases in optical lattices, Nat. Phys. 1, 23 (2005).

[17] M. Greiner, O. Mandel, T. Esslinger, T. W. Hänsch, and I. Bloch, Quantum phase transition from a superfluid to a Mott insulator in a gas of ultracold atoms, Nature (London) 415, 39 (2002).

[18] I. Bloch, J. Dalibard, and S. Nascimbène, Quantum simulations with ultracold quantum gases, Nat. Phys. 8, 267 (2012).

[19] C. Gross and I. Bloch, Quantum simulations with ultracold atoms in optical lattices, Science 357, 995 (2017).

[20] T. F. Gallagher, Rydberg Atoms (Cambridge University Press, Cambridge, UK, 1994).

[21] D. Jaksch, J. I. Cirac, P. Zoller, S. L. Rolston, R. Côté, and M. D. Lukin, Fast Quantum Gates for Neutral Atoms, Phys. Rev. Lett. 85, 2208 (2000).

[22] L. Santos, G. V. Shlyapnikov, P. Zoller, and M. Lewenstein, Bose-Einstein Condensation in Trapped Dipolar Gases, Phys. Rev. Lett. 85, 1791 (2000).

[23] C. H. Greene, A. S. Dickinson, and H. R. Sadeghpour, Creation of Polar and Nonpolar Ultra-Long-Range Rydberg Molecules, Phys. Rev. Lett. 85, 2458 (2000).

[24] W. R. Anderson, J. R. Veale, and T. F. Gallagher, Resonant Dipole-Dipole Energy Transfer in a Nearly Frozen Rydberg Gas, Phys. Rev. Lett. 80, 249 (1998).

[25] I. Mourachko, D. Comparat, F. de Tomasi, A. Fioretti, P. Nosbaum, V. M. Akulin, and P. Pillet, Many-Body Effects in a Frozen Rydberg Gas, Phys. Rev. Lett. 80, 253 (1998). 
[26] T. Wilk, A. Gaëtan, C. Evellin, J. Wolters, Y. Miroshnychenko, P. Grangier, and A. Browaeys, Entanglement of Two Individual Neutral Atoms Using Rydberg Blockade, Phys. Rev. Lett. 104, 010502 (2010).

[27] M. D. Lukin, M. Fleischhauer, R. Cote, L. M. Duan, D. Jaksch, J. I. Cirac, and P. Zoller, Dipole Blockade and Quantum Information Processing in Mesoscopic Atomic Ensembles, Phys. Rev. Lett. 87, 037901 (2001).

[28] J. Honer, H. Weimer, T. Pfau, and H. P. Büchler, Collective Many-Body Interaction in Rydberg Dressed Atoms, Phys. Rev. Lett. 105, 160404 (2010).

[29] Y.-Y. Jau, A. M. Hankin, T. Keating, I. H. Deutsch, and G. W. Biedermann, Entangling atomic spins with a Rydberg-dressed spin-flip blockade, Nat. Phys. 12, 71 (2016).

[30] J. Zeiher, R. van Bijnen, P. Schauss, S. Hild, J. yoon Choi, T. Pohl, I. Bloch, and C. Gross, Many-body interferometry of a Rydberg-dressed spin lattice, Nat. Phys. 12, 1095 (2016).

[31] J. B. Balewski, A. T. Krupp, A. Gaj, S. Hofferberth, R Löw, and T. Pfau, Rydberg dressing: Understanding of collective manybody effects and implications for experiments, New J. Phys. 16, 063012 (2014).

[32] F. Maucher, N. Henkel, M. Saffman, W. Królikowski, S. Skupin, and T. Pohl, Rydberg-Induced Solitons: ThreeDimensional Self-Trapping of Matter Waves, Phys. Rev. Lett. 106, 170401 (2011).

[33] J. Wang, J. N. Byrd, I. Simbotin, and R. Côté, Tuning Ultracold Chemical Reactions via Rydberg-Dressed Interactions, Phys. Rev. Lett. 113, 025302 (2014).

[34] A. W. Glaetzle, M. Dalmonte, R. Nath, C. Gross, I. Bloch, and P. Zoller, Designing Frustrated Quantum Magnets with LaserDressed Rydberg Atoms, Phys. Rev. Lett. 114, 173002 (2015).

[35] A. Arias, G. Lochead, T. M. Wintermantel, S. Helmrich, and S. Whitlock, Realization of a Rydberg-Dressed Ramsey Interferometer and Electrometer, Phys. Rev. Lett. 122, 053601 (2019).

[36] R. M. W. van Bijnen and T. Pohl, Quantum Magnetism and Topological Ordering via Rydberg Dressing near Förster Resonances, Phys. Rev. Lett. 114, 243002 (2015).

[37] M. Saffman, T. G. Walker, and K. Mølmer, Quantum information with Rydberg atoms, Rev. Mod. Phys. 82, 2313 (2010).

[38] A. K. Mohapatra, T. R. Jackson, and C. S. Adams, Coherent Optical Detection of Highly Excited Rydberg States Using Electromagnetically Induced Transparency, Phys. Rev. Lett. 98, 113003 (2007).

[39] C. Boisseau, I. Simbotin, and R. Côté, Macrodimers: Ultralong Range Rydberg Molecules, Phys. Rev. Lett. 88, 133004 (2002).

[40] N. Samboy, J. Stanojevic, and R. Côté, Formation and properties of Rydberg macrodimers, Phys. Rev. A 83, 050501(R) (2011).

[41] N. Samboy and R. Côté, Rubidium Rydberg macrodimers, J. Phys. B: At. Mol. Opt. Phys 44, 184006 (2011).

[42] K. R. Overstreet, A. Schwettmann, J. Tallant, D. Booth, and J. P. Shaffer, Observation of electric-field-induced Cs Rydberg atom macrodimers, Nat. Phys. 5, 581 (2009).

[43] H. Saßmannshausen and J. Deiglmayr, Observation of RydbergAtom Macrodimers: Micrometer-Sized Diatomic Molecules, Phys. Rev. Lett. 117, 083401 (2016).

[44] S. Hollerith, J. Zeiher, J. Rui, A. Rubio-Abadal, V. Walther, T. Pohl, D. M. Stamper-Kurn, I. Bloch, and C. Gross, Quantum gas microscopy of Rydberg macrodimers, Science 364, 664 (2019).
[45] E. Fermi, Sopra lo spostamento per pressione delle righe elevate delle serie spettrali, Nuovo Cimento 11, 157 (1934).

[46] A. A. Khuskivadze, M. I. Chibisov, and I. I. Fabrikant, Adiabatic energy levels and electric dipole moments of Rydberg states of $\mathrm{Rb}_{2}$ and $\mathrm{Cs}_{2}$ dimers, Phys. Rev. A 66, 042709 (2002).

[47] E. L. Hamilton, C. H. Greene, and H. R. Sadeghpour, Shape-resonance-induced long-range molecular Rydberg states, J. Phys. B: At. Mol. Opt. Phys. 35, L199 (2002).

[48] V. Bendkowsky, B. Butscher, J. Nipper, J. P. Shaffer, R. Löw, and T. Pfau, Observation of ultralong-range Rydberg molecules, Nature (London) 458, 1005 (2009).

[49] W. Li, T. Pohl, J. M. Rost, S. T. Rittenhouse, H. R. Sadeghpour, J. Nipper, B. Butscher, J. B. Balewski, V. Bendkowsky, R. Löw, and T. Pfau, A homonuclear molecule with a permanent electric dipole moment, Science 334, 1110 (2011).

[50] M. A. Bellos, R. Carollo, J. Banerjee, E. E. Eyler, P. L. Gould, and W. C. Stwalley, Excitation of Weakly Bound Molecules to Trilobitelike Rydberg States, Phys. Rev. Lett. 111, 053001 (2013).

[51] A. T. Krupp, A. Gaj, J. B. Balewski, P. Ilzhöfer, S. Hofferberth, R. Löw, T. Pfau, M. Kurz, and P. Schmelcher, Alignment of $d$ State Rydberg Molecules, Phys. Rev. Lett. 112, 143008 (2014).

[52] D. A. Anderson, S. A. Miller, and G. Raithel, Photoassociation of Long-Range nd Rydberg Molecules, Phys. Rev. Lett. 112, 163201 (2014).

[53] J. Tallant, S. T. Rittenhouse, D. Booth, H. R. Sadeghpour, and J. P. Shaffer, Observation of Blueshifted Ultralong-Range $\mathrm{Cs}_{2}$ Rydberg Molecules, Phys. Rev. Lett. 109, 173202 (2012).

[54] D. Booth, S. T. Rittenhouse, J. Yang, H. R. Sadeghpour, and J. P. Shaffer, Production of trilobite Rydberg molecule dimers with kilo-Debye permanent electric dipole moments, Science 348, 99 (2015).

[55] B. J. DeSalvo, J. A. Aman, F. B. Dunning, T. C. Killian, H. R. Sadeghpour, S. Yoshida, and J. Burgdörfer, Ultra-long-range Rydberg molecules in a divalent atomic system, Phys. Rev. A 92, 031403(R) (2015).

[56] N. Sándor, R. González-Férez, P. S. Julienne, and G. Pupillo, Rydberg optical Feshbach resonances in cold gases, Phys. Rev. A 96, 032719 (2017).

[57] O. Thomas, C. Lippe, T. Eichert, and H. Ott, Experimental realization of a Rydberg optical Feshbach resonance in a quantum many-body system, Nat. Commun. 9, 2238 (2018).

[58] M. T. Eiles and C. H. Greene, Hamiltonian for the inclusion of spin effects in long-range Rydberg molecules, Phys. Rev. A 95, 042515 (2017).

[59] C. Bahrim and U. Thumm, Low-lying ${ }^{3} P^{o}$ and ${ }^{3} S^{e}$ states of $\mathrm{Rb}^{-}$, $\mathrm{Cs}^{-}$, and $\mathrm{Fr}^{-}$, Phys. Rev. A 61, 022722 (2000).

[60] C. Bahrim, U. Thumm, and I. I. Fabrikant, Negative-ion resonances in cross sections for slow-electron-heavy-alkali-metalatom scattering, Phys. Rev. A 63, 042710 (2001).

[61] K. Bartschat and H. R. Sadeghpour, Ultralow-energy electron scattering from alkaline-earth atoms: The scattering-length limit, J. Phys. B: At. Mol. Opt. Phys. 36, L9 (2003).

[62] M. Aymar, C. H. Greene, and E. Luc-Koenig, Multichannel Rydberg spectroscopy of complex atoms, Rev. Mod. Phys. 68, 1015 (1996).

[63] S. M. Farooqi, D. Tong, S. Krishnan, J. Stanojevic, Y. P. Zhang, J. R. Ensher, A. S. Estrin, C. Boisseau, R. Côté, E. E. Eyler, and P. L. Gould, Long-Range Molecular Reso- 
nances in a Cold Rydberg Gas, Phys. Rev. Lett. 91, 183002 (2003).

[64] J. Stanojevic, R. Côté, D. Tong, E. E. Eyler, and P. L. Gould, Long-range potentials and $(n-1) d+n s$ molecular resonances in an ultracold Rydberg gas, Phys. Rev. A 78, 052709 (2008).

[65] J. Stanojevic, R. Côté, D. Tong, S. M. Farooqi, E. E. Eyler, and P. L. Gould, Long-range Rydberg-Rydberg interactions and molecular resonances, Eur. Phys. J. D 40, 3 (2006).

[66] J. E. Johnson and S. L. Rolston, Interactions between Rydbergdressed atoms, Phys. Rev. A 82, 033412 (2010).

[67] I. I. Beterov, I. I. Ryabtsev, D. B. Tretyakov, and V. M. Entin, Quasiclassical calculations of blackbody-radiation-induced depopulation rates and effective lifetimes of Rydberg $n s, n p$, and $n d$ alkali-metal atoms with $n \leqslant 80$, Phys. Rev. A 79, 052504 (2009).

[68] D. B. Branden, T. Juhasz, T. Mahlokozera, C. Vesa, R. O. Wilson, M. Zheng, A. Kortyna, and D. A. Tate, Radiative lifetime measurements of rubidium Rydberg states, J. Phys. B: At. Mol. Opt. Phys. 43, 015002 (2009).
[69] M. Schlagmüller, T. C. Liebisch, F. Engel, K. S. Kleinbach, F. Böttcher, U. Hermann, K. M. Westphal, A. Gaj, R. Löw, S. Hofferberth, T. Pfau, J. Pérez-Ríos, and C. H. Greene, Ultracold Chemical Reactions of a Single Rydberg Atom in a Dense Gas, Phys. Rev. X 6, 031020 (2016).

[70] J. Wang, H. Liu, G. Yang, B. Yang, and J. Wang, Determination of the hyperfine structure constants of the ${ }^{87} \mathrm{Rb}$ and ${ }^{85} \mathrm{Rb} 4 D_{5 / 2}$ state and the isotope hyperfine anomaly, Phys. Rev. A 90, 052505 (2014).

[71] S. Chandrasekhar, Stochastic problems in physics and astronomy, Rev. Mod. Phys. 15, 1 (1943).

[72] J. D. Whalen, S. K. Kanungo, R. Ding, M. Wagner, R. Schmidt, H. R. Sadeghpour, S. Yoshida, J. Bürgdorfer, F. B. Dunning, and T. C. Killian, Probing nonlocal spatial correlations in quantum gases with ultra-long-range Rydberg molecules, Phys. Rev. A 100, 011402 (2019).

[73] P. J. J. Luukko and J.-M. Rost, Polyatomic Trilobite Rydberg Molecules in a Dense Random Gas, Phys. Rev. Lett. 119, 203001 (2017). 